\title{
Space-time focusing of acoustic waves on unknown scatterers
}

\author{
Maxence Cassier and Christophe Hazard \\ ENSTA / POEMS ${ }^{1}, 32$ Boulevard Victor, 75015 Paris, France \\ (maxence.cassier@ensta-paristech.fr, christophe.hazard@ensta-paristech.fr)
}

January 24, 2014

\begin{abstract}
Consider a propagative medium, possibly inhomogeneous, containing some scatterers whose positions are unknown. Using an array of transmit-receive transducers, how can one generate a wave that would focus in space and time near one of the scatterers, that is, a wave whose energy would confine near the scatterer during a short time? The answer proposed in the present paper is based on the so-called DORT method (French acronym for: decomposition of the time reversal operator) which has led to numerous applications owing to the related space-focusing properties in the frequency domain, i.e., for time-harmonic waves. This method essentially consists in a singular value decomposition (SVD) of the scattering operator, that is, the operator which maps the input signals sent to the transducers to the measure of the scattered wave. By introducing a particular SVD related to the symmetry of the scattering operator, we show how to synchronize the time-harmonic signals derived from the DORT method to achieve space-time focusing. We consider the case of the scalar wave equation and we make use of an asymptotic model for small sound-soft scatterers, usually called the Foldy-Lax model. In this context, several mathematical and numerical arguments that support our idea are explored.
\end{abstract}

\section{Introduction}

\subsection{General context and objective}

Time reversal (TR) techniques have been extensively studied during the last decades, in particular owing to the related focusing properties. The simplest illustration of these properties consists in the following classical TR experiment (see, e.g., [9]). In a first step, an acoustic source emits a sound which propagates in the surrounding medium. The acoustic field is then measured and recorded by an array of transmitreceive transducers. In a second step, all the recordings are time-reversed and then used as input signals for the transducers of the array, generally called a time reversal mirror (TRM) because of this time reversal step. The acoustic wave emitted by the TRM propagates back in the same medium towards the original source. If this source fills a small region and if the initial sound is a short pulse, one can observe that a great part of the acoustic energy of the back-propagating wave will be concentrated near the original source at a given time. In this sense, space-time focusing is achieved. The focus quality will be high if the TRM surrounds completely the source (full aperture case) and if the number of transducers is large enough. On the other hand, the focusing will generally deteriorate if the number of transducers becomes smaller or if the aperture of the TRM is reduced, that is, if the angular area occupied by the TRM decreases (partial aperture case).

In such a TR experiment, the source is active in the sense that it emits the original sound which is then recorded by the TRM. In the present paper, we are no longer concerned by active sources, but rather by unknown scatterers which behave passively only by their ability to reflect an incident wave. Hence the way to retrieve some information on the scatterers is to test the medium by means of the array of

\footnotetext{
${ }^{1}$ POEMS (Propagation d'Ondes: Etude Mathématique et Simulation) is a mixed research team (UMR 7231) between CNRS (Centre National de la Recherche Scientifique), ENSTA ParisTech (Ecole Nationale Supérieure de Techniques Avancées) and INRIA (Institut National de Recherche en Informatique et en Automatique).
} 
transducers which can emit various incident waves and record the response of the medium. We assume here that we are able to measure the scattered wave, that is, the perturbation of the incident wave due to the presence of the scatterers. This means implicitly that we are able to compare the propagation of an acoustic wave in two different media: on the one hand, a reference medium which is the surrounding medium in the absence of the scatterers, on the other hand a perturbed medium which is the same medium perturbed by the presence of the scatterers. In other words, each test of the medium consists actually in two experiments: for given input signals sent to the transducers, the same incident wave is emitted by the array in both reference and perturbed media, both responses are then measured by the array, and finally the former is subtracted from the latter. The operator which maps the input signals to the difference of both measures will be called here the scattering operator (it is often referred to as the multi-static response matrix). The question we are interested in is the following: from the only knowledge of this scattering operator, can one generate a wave which would focus in both space and time at one of the scatterers? There is no doubt that a positive answer to this question could lead to numerous applications in nondestructive testing, medicine, communication, imaging, etc.

The method we propose is based on the so-called DORT method (French acronym for: decomposition of the time reversal operator) first developed by Prada and Fink [19, 21, 22] in the context of ultrasonics. Numerous experimental, numerical and theoretical applications of this method have been explored for acoustic, elastic or electromagnetic waves. The DORT method can be seen as an improvement of iterative time reversal in the frequency domain. It is based actually on a singular value decomposition (SVD) of the above mentioned scattering operator for time-harmonic waves. It is now well understood that for distant enough and small scatterers, the number of non-negligible singular values of the scattering operator coincide with the number of scatterers. Moreover, when these singular values are simple, each corresponding singular vector generates a wave which focuses selectively on each scatterer. These properties were first observed for point-like targets [21, 22] and then confirmed by different theoretical studies (see, e.g., [12] for acoustic waves and [1] for electromagnetic waves). Numerical results [2] show that these selective focusing properties hold even when the asymptotic conditions are not satisfied, that is, for rather close scatterers which are not so small in comparison with the wavelength.

In short, the DORT method provides us spatial selective focusing in the frequency domain. This is the starting point of the present paper which addresses the following issue: can we take advantage of these spatial focusing properties in the frequency domain in order to produce a time-dependent wave which would also focus in time? This challenging question was first raised in [17]. It mainly consists in a phase synchronization issue, since the singular vectors of the scattering operator are defined up to a phase shift. The same issue was also mentioned in [4] which presents an extension to the time domain of the so-called MUSIC algorithm (multiple signal classification) for imaging in random media. To our knowledge, the first proposal of solution was given in [18] using an approximation by a homogeneous free-space back propagation. The method we propose does not involve any approximation. It is based on the symmetry of the scattering operator which allows us to choose a particular SVD, called the symmetric singular value decomposition (SSVD). The idea of using this particular SVD is not really new: we discovered recently that it is briefly mentioned in an unpublished report [20], but with no justification. Our aim is to explore several arguments which show that the use of the SSVD of the scattering operator yields space-time focusing.

The paper is organized as follows. We begin by describing in $\S 1.2$ the mathematical formulation of the problem and we introduce the main notations which are used throughout the paper. Section 2 is devoted to space focusing in the frequency domain. As the focusing properties of the DORT method hold for small scatterers, we make use of an asymptotic model, usually called the Foldy-Lax model, which considers point-like scatterers. In this context, we briefly recall the main features of the DORT method and we illustrate them by numerical results. Section 3 constitutes the core of the paper. We first show in $\S 3.1$ how to reduce the problem to a phase synchronization issue and introduce the SSVD of the scattering operator. We then prove in $\S 3.2$ that the choice of this SSVD is associated with the optimum of a TR criterion. In $\S 3.3$, we explore the link between the SSVD and the TR experiment mentioned above. Finally the numerical results of $\$ 3.4$ illustrate the space-time focusing effect. The focus quality is evaluated by means of an energy criterion which involves some technical calculations that are collected in the Appendix. 


\subsection{Mathematical formulation of the problem}

In the present paper, we consider a reference medium, possibly inhomogeneous in a bounded region, filling the whole space $\mathbb{R}^{d}$ (with $d>1$ ). This medium is characterized by a sound velocity function $c=c(x)$ for $x \in \mathbb{R}^{d}$. We denote by $G=G(x, y, t)$ the time-dependent Green's function of the acoustic wave equation, which is solution to

$$
\frac{1}{c^{2}(x)} \frac{\partial^{2} G(x, y, t)}{\partial t^{2}}-\Delta_{x} G(x, y, t)=\delta(x-y) \otimes \delta(t),
$$

where $\delta$ stands for the Dirac measure at the origin, either in space or time. Moreover $G$ is assumed to be causal in the sense that $G(x, y, t)=0$ for all $(x, y) \in \mathbb{R}^{2 d}$ and $t<0$ (which ensures the uniqueness of $G)$. Recall that for a homogeneous medium, i.e., $c \equiv 1$, this function is given by

$$
G_{\text {hom }}(x, y, t)= \begin{cases}\frac{H(t-|x-y|)}{2 \pi \sqrt{t^{2}-|x-y|^{2}}} & \text { if } d=2, \\ \frac{\delta(t-|x-y|)}{4 \pi|x-y|} & \text { if } d=3,\end{cases}
$$

where $H$ is the Heaviside function.

We consider an array of $N$ point-like transducers located at $x_{n}$ for $n=1, \ldots, N$ which can emit incident waves of the form

$$
v_{\text {inc }}(x, t):=\sum_{n=1}^{N}\left(q_{n} \stackrel{t}{\star} G\left(x, x_{n}, \cdot\right)\right)(t)=\sum_{n=1}^{N} \int_{\mathbb{R}} q_{n}\left(t^{\prime}\right) G\left(x, x_{n}, t-t^{\prime}\right) \mathrm{d} t^{\prime}
$$

where $\boldsymbol{q}_{\text {in }}(t):=\left(q_{1}(t), \ldots, q_{N}(t)\right)^{\top}$ represents the vector composed of the input signals which are applied to the transducers (the symbol $\top$ indicates transposition) and $\stackrel{t}{\star}$ denotes the convolution with respect to time.

We suppose that our reference medium is perturbed by the presence of a family of $P$ sound-soft scatterers $\mathcal{S}_{p} \subset \mathbb{R}^{d}$ for $p=1, \ldots, P$. In this case, when the same signals $\boldsymbol{q}_{\mathrm{in}}(t)$ are sent to the transducers, we observe instead of the incident wave (2) a perturbed wave $v_{\text {inc }}+v_{\mathrm{sc}}$ where $v_{\mathrm{sc}}$ represents the scattered wave, that is, the part of the wave due to the presence of the scatterers, which is the causal solution to

$$
\begin{aligned}
& \frac{1}{c^{2}(x)} \frac{\partial^{2} v_{\mathrm{sc}}}{\partial t^{2}}-\Delta v_{\mathrm{sc}}=0 \quad \text { in } \mathbb{R}^{d} \backslash \cup_{p=1}^{P} \mathcal{S}_{p}, \\
& v_{\mathrm{sc}}=-v_{\text {inc }} \quad \text { on } \cup_{p=1}^{P} \partial \mathcal{S}_{p} .
\end{aligned}
$$

This wave is finally measured by the transducers, which yields a vector composed of $N$ functions which is denoted by $\boldsymbol{q}_{\text {out }}(t):=\left(v_{\mathrm{sc}}\left(x_{1}, t\right), \ldots, v_{\mathrm{sc}}\left(x_{N}, t\right)\right)^{\top}$. The linear operator

$$
\mathbb{S}: \boldsymbol{q}_{\text {in }} \longmapsto \boldsymbol{q}_{\text {out }}
$$

is called here the scattering operator. Note that this definition differs slightly from the scattering operator involved in mathematical physics.

In this paper, we use a spectral representation of $\mathbb{S}$, which amounts to representing all time-dependent signals as superpositions of time-harmonic signals. Thus we introduce the Fourier transform $\mathcal{F}$ with respect to time defined by

$$
\hat{f}(\omega):=\mathcal{F} f(\omega):=\int_{-\infty}^{+\infty} f(t) \mathrm{e}^{+\mathrm{i} \omega t} \mathrm{~d} t
$$

whose inverse is given by

$$
f(t)=\mathcal{F}^{-1} \hat{f}(t)=\frac{1}{2 \pi} \int_{-\infty}^{+\infty} \hat{f}(\omega) \mathrm{e}^{-\mathrm{i} \omega t} \mathrm{~d} \omega .
$$


Note that in the case where $f$ is real-valued, its Fourier transform satisfies the symmetry property

$$
f(-\omega)=\overline{f(\omega)} \text { so that } f(t)=\frac{1}{\pi} \operatorname{Re} \int_{0}^{+\infty} \hat{f}(\omega) \mathrm{e}^{-\mathrm{i} \omega t} \mathrm{~d} \omega .
$$

Define $\hat{\boldsymbol{q}}_{\text {in }}(\omega):=\mathcal{F} \boldsymbol{q}_{\text {in }}(\omega)=\left(\hat{q}_{1}(\omega), \ldots, \hat{q}_{N}(\omega)\right)^{\top}$. For all $\omega, \hat{\boldsymbol{q}}_{\text {in }}(\omega)$ represents the time-harmonic input signals which emit a time-harmonic incident wave

$$
\hat{v}_{\text {inc }}(x, \omega):=\left(\mathcal{F} v_{\text {inc }}(x, \cdot)\right)(\omega)=\sum_{n=1}^{N} \hat{q}_{n}(\omega) \hat{G}\left(x, x_{n}, \omega\right),
$$

where $\hat{G}=\hat{G}(x, y, \omega)$ denotes the Fourier transform of $G$, that is, the time-harmonic Green's function of the reference medium. Its expression is well-known for a homogeneous medium $(c \equiv 1)$ :

$$
\hat{G}_{\mathrm{hom}}(x, y, \omega)= \begin{cases}\frac{\mathrm{i}}{4} H_{0}^{(1)}(\omega|x-y|) & \text { if } d=2, \\ \frac{\mathrm{e}^{\mathrm{i} \omega|x-y|}}{4 \pi|x-y|} & \text { if } d=3,\end{cases}
$$

where $H_{0}^{(1)}$ is the Hankel function of the first kind and order 0 . The interaction of $\hat{v}_{\text {inc }}(x, \omega)$ with the scatterers produces a scattered wave $\hat{v}_{\mathrm{sc}}(x, \omega)=\left(\mathcal{F} v_{\mathrm{sc}}(x, \cdot)\right)(\omega)$ which is finally measured by the transducers. This yields the time-harmonic scattering operator

$$
\widehat{\mathbb{S}}_{\omega}: \hat{\boldsymbol{q}}_{\text {in }}(\omega) \longmapsto \hat{\boldsymbol{q}}_{\text {out }}(\omega):=\left(\hat{v}_{\mathrm{sc}}\left(x_{1}, \omega\right), \ldots, \hat{v}_{\mathrm{sc}}\left(x_{N}, \omega\right)\right)^{\top} .
$$

We can sum up the link between the time-dependent and time-harmonic scattering operators by the equality

$$
\mathbb{S}=\mathcal{F}^{-1} \widehat{\mathbb{S}} \mathcal{F}
$$

where $\widehat{\mathbb{S}}$ denotes the operator defined from the family $\left\{\widehat{\mathbb{S}}_{\omega} ; \omega \in \mathbb{R}\right\}$ by the simple relation

$$
(\widehat{\mathbb{S}} \hat{\boldsymbol{q}})(\omega)=\widehat{\mathbb{S}}_{\omega}(\hat{\boldsymbol{q}}(\omega)) .
$$

The question we address in the present paper is the following: from the only knowledge of the operator $\mathbb{S}$, how can one generate a wave that focuses selectively in space and time on one of the scatterers? The answer we propose is the subject of section 3. It is based on the DORT method presented in the next section.

\section{Space focusing in the frequency domain}

\subsection{The scattering operator for small scatterers}

As mentioned in the introduction, the spatial focusing properties related to the DORT method hold for small enough scatterers. That is why we use in the sequel an asymptotic model in the frequency domain, which enables us to replace the family $\left\{\mathcal{S}_{p} \subset \mathbb{R}^{d} ; p=1, \ldots, P\right\}$ by a family of $P$ point-like scatterers located at $y_{1}, \ldots, y_{P}$. The model we use, often called the Foldy-Lax model [10], has the advantage to take into account an approximation of the interactions between the scatterers. It is widely used by physicists. For instance, [16] presents an application of the MUSIC method for estimating the locations and reflectivities of point-like inhomogeneities. A mathematical justification of the Foldy-Lax model was recently proposed in [7] for the two-dimensional problem.

This model is based on the fact that in the case of one single sound-soft scatterer $(P=1)$ whose size is small compared to the wavelength, the time-harmonic scattered field $\hat{v}_{\mathrm{sc}}$ behaves like the field emitted by a point source (see [15]). More precisely, one has

$$
\hat{v}_{\mathrm{sc}}(x, \omega) \approx \sigma(\omega) \hat{v}_{\mathrm{inc}}(y, \omega) \hat{G}(x, y, \omega),
$$


where $y \in \mathbb{R}^{d}$ is the location of the scatterer and $\sigma(\omega) \in \mathbb{C}$ is its reflectivity coefficient. For instance, in a homogeneous medium $(c \equiv 1)$, if the scatterer is circular $(d=2)$ or spherical $(d=3)$ with radius $\varepsilon$, we have

$$
\sigma(\omega)= \begin{cases}-4 i / H_{0}^{(1)}(\omega \varepsilon) & \text { if } d=2 \\ 4 \pi \varepsilon & \text { if } d=3\end{cases}
$$

For several obstacles $(P>1)$, the scattered field appears as a superposition of similar behaviours,

$$
\hat{v}_{\mathrm{sc}}(x, \omega) \approx \sum_{p=1}^{P} \sigma_{p}(\omega) \hat{v}_{p}(\omega) \hat{G}\left(x, y_{p}, \omega\right)
$$

where $\hat{v}_{p}(\omega)$ represents the exciting field on the $p$-th scatterer. Choosing $\hat{v}_{p}(\omega)=\hat{v}_{\text {inc }}\left(y_{p}, \omega\right)$ as for a single scatterer amounts to neglecting the interactions between the scatterers. The Foldy-Lax model consists in correcting the latter choice by adding the waves scattered by all the other obstacles, i.e.,

$$
\hat{v}_{p}(\omega)=\hat{v}_{\text {inc }}\left(y_{p}, \omega\right)+\sum_{q \neq p} \sigma_{q}(\omega) \hat{v}_{q}(\omega) \hat{G}\left(y_{p}, y_{q}, \omega\right) \quad \text { for } p=1, \ldots, P .
$$

If we denote by $\widehat{V}_{\omega}$ and $\widehat{V}_{\omega}^{\text {inc }}$ the vectors of $\mathbb{C}^{P}$ with components $\hat{v}_{p}(\omega)$ and $\hat{v}_{\text {inc }}\left(y_{p}, \omega\right)$ respectively, this coupling between the exciting fields can be written equivalently as

$$
\left(\mathbb{I}-\widehat{\mathbb{H}}_{\omega} \Sigma_{\omega}\right) \widehat{V}_{\omega}=\widehat{V}_{\omega}^{\text {inc }},
$$

where $\Sigma_{\omega}$ is the $P \times P$ diagonal matrix composed of the reflectivity coefficients, i.e., $\left(\Sigma_{\omega}\right)_{p p}:=\sigma_{p}(\omega)$, and $\widehat{\mathbb{H}}_{\omega}$ is the $P \times P$ matrix defined by

$$
\left(\widehat{\mathbb{H}}_{\omega}\right)_{p q}:=\hat{G}\left(y_{p}, y_{q}, \omega\right) \text { if } q \neq p \quad \text { and } \quad\left(\widehat{\mathbb{H}}_{\omega}\right)_{p p}:=0 \text {. }
$$

The use of the Foldy-Lax model yields a simple expression for the time-harmonic scattering operator (7). Indeed, applying (10) to $x=x_{n}$ for $n=1$ to $N$ yields

$$
\hat{\boldsymbol{q}}_{\mathrm{out}}(\omega)=\widehat{\mathbb{G}}_{\omega}^{\top} \Sigma_{\omega} \widehat{V}_{\omega}
$$

where $\widehat{\mathbb{G}}_{\omega}$ denotes the $P \times N$ matrix defined by

$$
\left(\widehat{\mathbb{G}}_{\omega}\right)_{p n}:=\hat{G}\left(x_{n}, y_{p}, \omega\right)
$$

and we recall that the superscript $\top$ indicates transposition. Moreover, noticing from (6) that the righthand side of (11) writes as $\widehat{V}_{\omega}^{\text {inc }}=\widehat{\mathbb{G}}_{\omega} \hat{\boldsymbol{q}}_{\text {in }}(\omega)$, we have $\widehat{V}_{\omega}=\left(\mathbb{I}-\widehat{\mathbb{H}}_{\omega} \Sigma_{\omega}\right)^{-1} \widehat{\mathbb{G}}_{\omega} \hat{\boldsymbol{q}}_{\text {in }}(\omega)$. Substituting this expression in (12) yields finally

$$
\widehat{\mathbb{S}}_{\omega}=\widehat{\mathbb{G}}_{\omega}^{\top}\left(\Sigma_{\omega}^{-1}-\widehat{\mathbb{H}}_{\omega}\right)^{-1} \widehat{\mathbb{G}}_{\omega} .
$$

This product of three matrices can be interpreted as the succession of three steps in the scattering process. First $\widehat{\mathbb{G}}_{\omega}$ represents the propagation of the incident wave from the transducers to the scatterers. Then $\left(\Sigma_{\omega}^{-1}-\widehat{\mathbb{H}}_{\omega}\right)^{-1}$ describes the reflection on the scatterers (note that this matrix reduces to $\Sigma_{\omega}$ if $\widehat{\mathbb{H}}_{\omega}$ is neglected, which amounts to neglecting the interaction between the scatterers). And finally $\widehat{\mathbb{G}}_{\omega}^{\top}$ corresponds to back-propagation from the scatterers to the transducers.

Remark 1. It is readily seen that the scattering operator is symmetric (but not hermitian) in the sense that $\widehat{\mathbb{S}}_{\omega}=\widehat{\mathbb{S}}_{\omega}^{\top}$ (but $\widehat{\mathbb{S}}_{\omega} \neq \widehat{\mathbb{S}}_{\omega}^{*}$ where $\widehat{\mathbb{S}}_{\omega}^{*}$ is the adjoint matrix, i.e., the conjugate transpose matrix) since both matrices $\Sigma_{\omega}$ and $\widehat{\mathbb{H}}_{\omega}$ are symmetric. This can be seen as a consequence of the reciprocity principle. 


\subsection{The DORT method}

The DORT method $[19,21,22]$ consists in a singular value decomposition (SVD) of the scattering operator $\widehat{\mathbb{S}}_{\omega}$ :

$$
\widehat{\mathbb{S}}_{\omega}=\widehat{\mathbb{V}}_{\omega} \widehat{\mathbb{D}}_{\omega} \widehat{\mathbb{W}}_{\omega}^{*}
$$

where $\widehat{\mathbb{D}}_{\omega}$ is a $N \times N$ diagonal matrix with nonnegative real numbers on the diagonal, whereas $\widehat{\mathbb{V}}_{\omega}$ and $\widehat{\mathbb{W}}_{\omega}$ are unitary $N \times N$ matrices. The diagonal entries of $\widehat{\mathbb{D}}_{\omega}$ are known as the singular values of $\widehat{\mathbb{S}}_{\omega}$ and the columns of $\widehat{\mathbb{V}}_{\omega}$ and $\widehat{\mathbb{W}}_{\omega}$ are called the left and right singular vectors, respectively.

A real number $\lambda \geq 0$ is a singular value of $\widehat{\mathbb{S}}_{\omega}$ if and only if there exist unit vectors $\hat{\boldsymbol{v}}$ and $\hat{\boldsymbol{w}}$ in $\mathbb{C}^{N}$ such that

$$
\widehat{\mathbb{S}}_{\omega} \hat{\boldsymbol{w}}=\lambda \hat{\boldsymbol{v}} \quad \text { and } \quad \widehat{\mathbb{S}}_{\omega}^{*} \hat{\boldsymbol{v}}=\lambda \hat{\boldsymbol{w}}
$$

Vectors $\hat{\boldsymbol{v}}$ and $\hat{\boldsymbol{w}}$ are respectively left and right singular vectors of $\widehat{\mathbb{S}}_{\omega}$.

Remark 2. The SVD of $\widehat{\mathbb{S}}_{\omega}$ is not unique (actually $\widehat{\mathbb{D}}_{\omega}$ is unique but not $\widehat{\mathbb{V}}_{\omega}$ and $\widehat{\mathbb{W}}_{\omega}$ ). This is obvious if at least one eigenvalue is degenerate, that is, if the dimension of the associated space of right (respectively, left) singular vectors is greater than one. On the other hand, for a simple (or non-degenerate) singular value, the right and left singular vectors are unique up to a multiplication by a unit complex number. Indeed it is readily seen that if a pair $(\hat{\boldsymbol{v}}, \hat{\boldsymbol{w}})$ satisfies $(15)$, then so does $\left(\mathrm{e}^{\mathrm{i} \phi} \hat{\boldsymbol{v}}, \mathrm{e}^{\mathrm{i} \phi} \hat{\boldsymbol{w}}\right)$ for all $\phi \in \mathbb{R}$.

Remark 3. The singular values of $\widehat{\mathbb{S}}_{\omega}$ are the square roots of the eigenvalues of the positive hermitian matrix $\widehat{\mathbb{S}}_{\omega}^{*} \widehat{\mathbb{S}}_{\omega}$ and the right singular vectors of $\widehat{\mathbb{S}}_{\omega}$ coincide with the eigenvectors of $\widehat{\mathbb{S}}_{\omega}^{*} \widehat{\mathbb{S}}_{\omega}$. The initial presentation of the DORT method (see, e.g., [21, 22]) consisted in the diagonalization of the latter matrix, called the time reversal operator because it was derived from an iterative TR process. The basic loop of this process consists of the following steps: for given input signals $\hat{\boldsymbol{q}}$, the array of transducers first emit an incident wave; the associated scattered wave in then measured, which yields $\widehat{\mathbb{S}}_{\omega} \hat{\boldsymbol{q}}$; and finally this measure is time-reversed, which amounts to a conjugation in the frequency domain (see (21)). The time-reversed measure can then be used to re-emit a new incident wave. The time reversal operator $\widehat{\mathbb{T}}_{\omega}$ describes two successive iterations of this loop, that is,

$$
\widehat{\mathbb{T}}_{\omega} \hat{\boldsymbol{q}}:=\overline{\widehat{\mathbb{S}}_{\omega} \widehat{\mathbb{S}}_{\omega} \hat{\boldsymbol{q}}}=\overline{\widehat{\mathbb{S}}_{\omega}} \widehat{\mathbb{S}}_{\omega} \hat{\boldsymbol{q}} \quad \text { so } \quad \widehat{\mathbb{T}}_{\omega}=\widehat{\mathbb{S}}_{\omega} \widehat{\mathbb{S}}_{\omega}=\widehat{\mathbb{S}}_{\omega}^{*} \widehat{\mathbb{S}}_{\omega}
$$

where the last equality follows from the symmetry of $\widehat{\mathbb{S}}_{\omega}$.

The main interest of the DORT method lies in the selective focusing properties related to the singular vectors of $\widehat{\mathbb{S}}_{\omega}$. In our context of point-like scatterers, it is well understood $[12,21,22]$ that if the reference medium is homogeneous and if the scatterers are distant enough from each other (they are often referred to as ideally resolved in this case), then

1. the number of scatterers $P$ is equal to the number of nonzero singular values of $\widehat{\mathbb{S}}_{\omega}$ (provided that $P \leq N$ and that the rank of $\widehat{\mathbb{G}}_{\omega}$ is equal to $P$, which excludes some exceptional situations such as scatterers located symmetrically with respect to the array);

2. when these singular values are simple, each right singular vector (column of $\widehat{\mathbb{W}}_{\omega}$ ) associated with each nonzero singular value generates a wave which focuses selectively on each scatterer.

Figures 1 to 3 illustrate these results by some numerical experiments in the two-dimensional case. We first consider the case of a homogeneous medium (i.e., $c \equiv 1$ ) filling the whole plane $\mathbb{R}^{2}$. The part of the plane represented on each figure is the square $[0,2] \times[0,2]$. Our array is composed of 128 transducers $(N=128)$ regularly distributed along the segment $\{0\} \times[0,2]$ which corresponds to the left edge of each figure. Two scatterers $(P=2)$, with respective diameters $2 \times 10^{-3}$ and $10^{-3}$, are located at $(1.2,0.3)$ and $(1.7,1.9)$ respectively. They are represented by white disks (not to scale). The time-harmonic scattering operator has two non-zeros singular values in this case. For the circular frequency $\omega=40$, that is, a wavelength $\lambda=2 \pi / \omega \approx 0.16$, Figure 1 shows the modulus of the incident field generated by the associated 

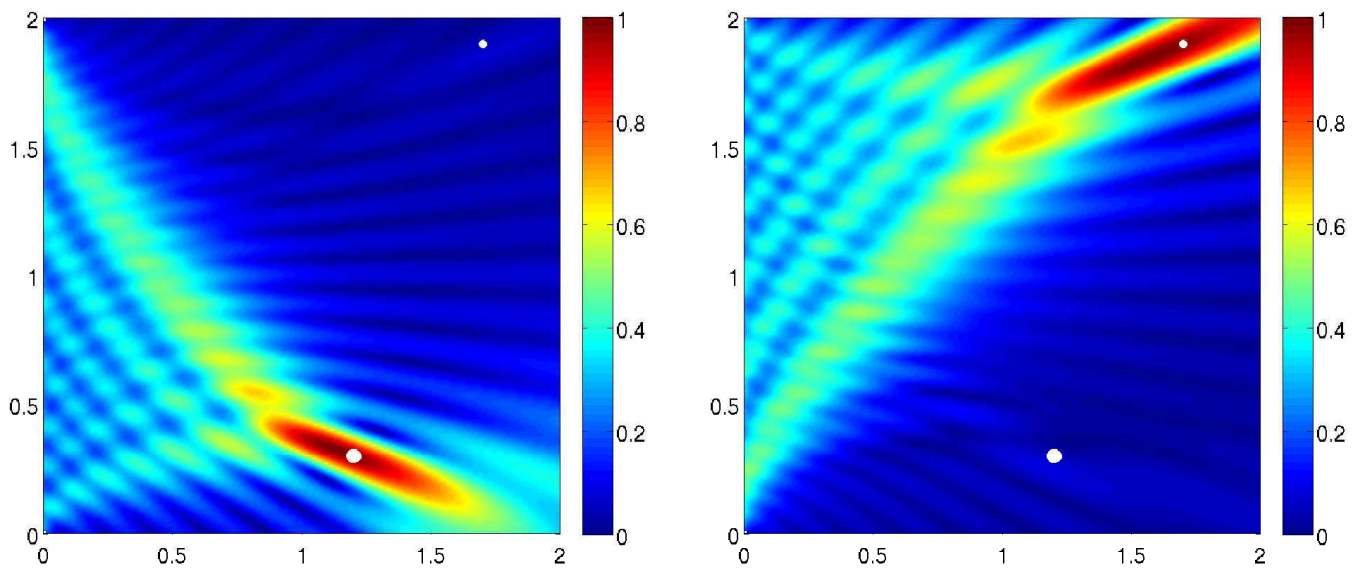

Figure 1: Modulus of the incident field generated by the right singular vectors of $\widehat{\mathbb{S}}_{\omega}$ associated with the dominant (left) and second (right) singular values. Case of a homogeneous medium with $\omega=40$.
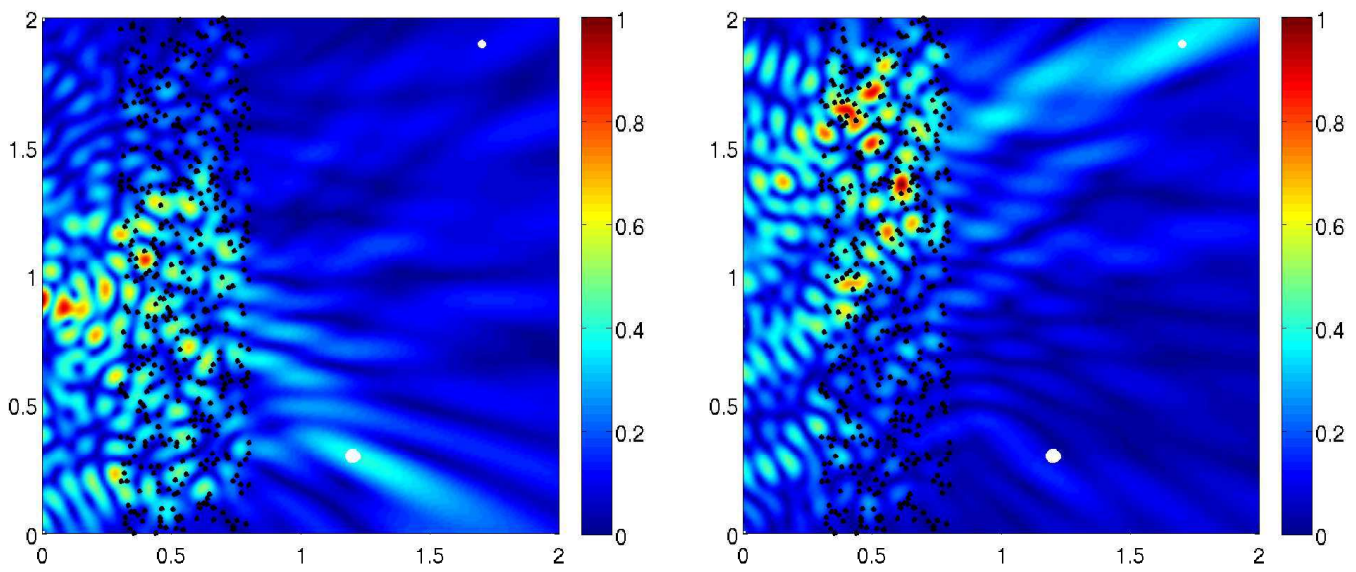

Figure 2: Same as Figure 1 in the case of a scattering medium with $\omega=40$.
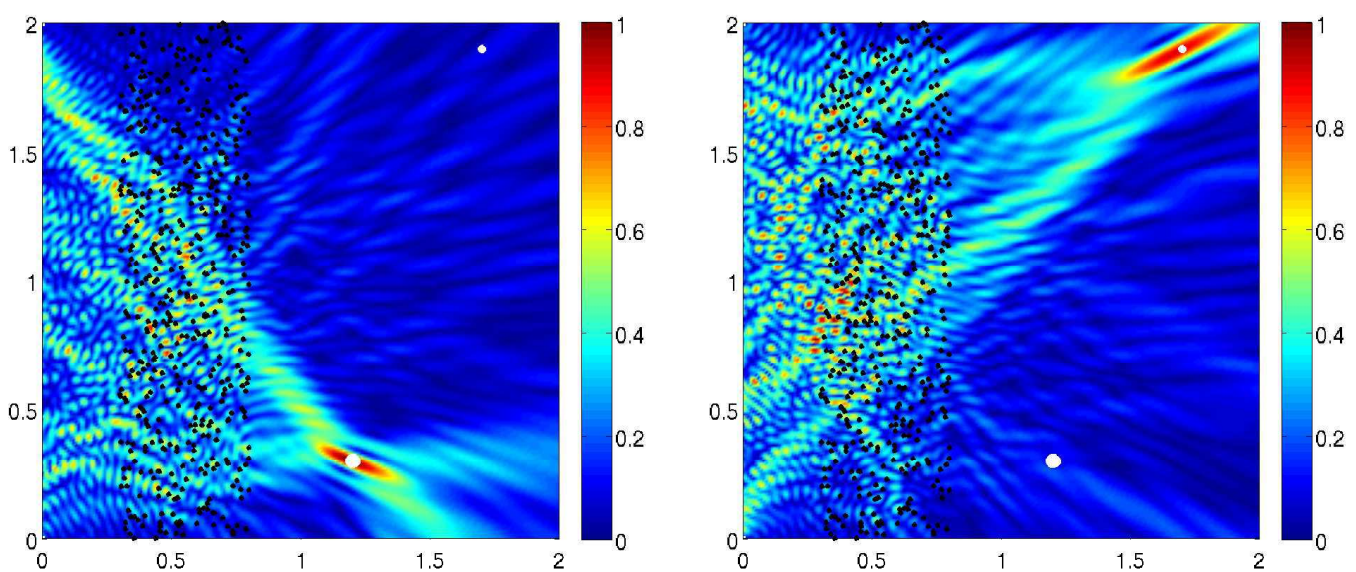

Figure 3: Same as Figure 2 for $\omega=90$. 
singular vectors. We clearly see the spatial focusing effect: the field becomes maximal in an elliptical area centered at each scatterer, generally called the focal spot. If $\ell$ denotes the length of the array (here $\ell=2$ ) and $d$ is the distance between the array and the considered scatterer, the transverse radius of this area is given by $\lambda d / \ell$ (Rayleigh resolution formula, see, e.g., [6]) provided that $\lambda \ll \ell \ll d$. For instance $\lambda d / \ell \approx 0,1$ for the biggest scatterer, which is consistent with the observed focal spot though the assumption $\ell \ll d$ is not fulfilled.

In Figures 2 and 3, we consider the case where a scattering medium is located between the array and the scatterers. Here our scattering medium consists of 500 point-like scatterers with same diameter $2 \times 10^{-4}$ (represented by black points) randomly displayed in the rectangle $[0.3,0.8] \times[0,2]$. In this situation, the Green's function of the reference medium appears as a perturbation of the Green's function of the homogeneous medium:

$$
\hat{G}(x, y, \omega)=\hat{G}_{\mathrm{hom}}(x, y, \omega)+\hat{G}_{\mathrm{sc}}(x, y, \omega)
$$

where the perturbation $\hat{G}_{\mathrm{Sc}}(x, y, \omega)$ can be computed by the Foldy-Lax model described in section 2.1, which requires us to invert the $500 \times 500$ matrix involved in equation (11). Figure 2 represents again the modulus of the incident field generated by the singular vectors associated with the non-zero eigenvalues of $\widehat{\mathbb{S}}_{\omega}$ for the same circular frequency $\omega=40$ as in Figure 1 . We see that the presence of the scattering medium has damaged considerably the spatial focusing effect. Focal spots are still observed, but their intensity is reduced significantly, for the acoustic field seems to be trapped in the scattering medium. Figure 3 shows that for the higher circular frequency $\omega=90$, spatial focusing is recovered.

The physical notions of ballistic and diffusive regimes (see, e.g., [8]) can help us to interpret these numerical observations. We have to compare the thickness $L$ of the scattering medium with the mean free path which is given here by

$$
L_{\mathrm{fp}}:=\frac{4 \omega}{\mathcal{N}|\sigma(\omega)|^{2}}
$$

where $\mathcal{N}$ is the number of scatterers per unit surface and $\sigma(\omega)$ is the reflectivity coefficient defined in (9). The so-called ballistic regime corresponds to the case where $L \ll L_{\mathrm{fp}}$. In such a situation, when a plane wave crosses the scattering medium, its direction of propagation is not much affected and the perturbation of the plane wave essentially consists in the specular reflections on the scatterers. Higher order successive reflections are negligible. On the other hand, when $L \gg L_{\mathrm{fp}}$, the latter may become significant, which leads to resonant phenomena inside the scattering medium. In such a diffusive regime, the structure of a wave crossing the medium is strongly perturbed. In the case of Figure $2(\omega=40)$, we have $L_{\mathrm{fp}} \approx 0.28$ whereas $L=0.5$, which rather corresponds to the diffusive regime. In the case of Figure $3(\omega=90)$, we have $L_{\mathrm{fp}} \approx 0.47$, which corresponds to an intermediate regime. In particular, resonances are still observed in the scattering medium. By increasing the frequency further, we would enter the ballistic regime and the focus quality would improve.

\section{Space-time focusing}

\subsection{From frequency domain to time domain}

\section{A phase synchronization issue.}

We arrive now at the main purpose of the paper, which is to take advantage of the selective focusing properties of the DORT method in the frequency domain in order to construct a time-dependent wave which focuses in space and time on one given scatterer chosen as a target. Suppose that for all frequency $\omega$ in a given frequency band $\left[\omega_{1}, \omega_{2}\right]$ (which depends on the specifications of the transducers), we know a triplet $(\lambda(\omega), \hat{\boldsymbol{v}}(\omega), \hat{\boldsymbol{w}}(\omega))$ of singular elements of the scattering operator corresponding to our target: $\lambda(\omega)>0$ is a singular value, which is assumed simple for simplicity, and the associated left and right singular vectors $\hat{\boldsymbol{v}}(\omega)$ and $\hat{\boldsymbol{w}}(\omega)$ are unit vectors of $\mathbb{C}^{N}$ such that

$$
\widehat{\mathbb{S}}_{\omega} \hat{\boldsymbol{w}}(\omega)=\lambda(\omega) \hat{\boldsymbol{v}}(\omega) \quad \text { and } \quad \widehat{\mathbb{S}}_{\omega}^{*} \hat{\boldsymbol{v}}(\omega)=\lambda(\omega) \hat{\boldsymbol{w}}(\omega)
$$


The fact that these singular elements correspond to our target means that the time-harmonic wave generated by the right singular vector $\hat{\boldsymbol{w}}(\omega)$ focuses spatially on this target. As this spatial focusing occurs for all $\omega \in\left[\omega_{1}, \omega_{2}\right]$, it holds true for any frequency superposition of these waves. So if we consider a pair $(\chi, \phi)$ of real-valued functions defined on $\left[\omega_{1}, \omega_{2}\right]$, the time-dependent input signal defined by

$$
\boldsymbol{q}_{\phi}(t):=\frac{1}{\pi} \operatorname{Re} \int_{\omega_{1}}^{\omega_{2}} \chi(\omega) \mathrm{e}^{\mathrm{i} \phi(\omega)} \hat{\boldsymbol{w}}(\omega) \mathrm{e}^{-\mathrm{i} \omega t} \mathrm{~d} \omega
$$

generates a time-dependent wave which focuses in space on our target. But can we choose these functions so that the above superposition of time-harmonic signals yields also time focusing? Instead of searching for a pair $(\chi, \phi)$, we assume here that $\chi$ is known and we restrict ourselves to the determination of the phase function $\phi:\left[\omega_{1}, \omega_{2}\right] \mapsto \mathbb{R}$ (which justifies the fact that $\chi$ is not involved in the notation $\boldsymbol{q}_{\phi}$ above). In other words, the issue we are faced with is to synchronize the singular vectors of $\widehat{\mathbb{S}}_{\omega}$ in order to achieve space-time focusing (recall that $\mathrm{e}^{\mathrm{i} \phi(\omega)} \hat{\boldsymbol{w}}(\omega)$ is a right singular vector of $\widehat{\mathbb{S}}_{\omega}$ whatever $\phi(\omega)$, see remark 2).

\section{Continuity assumptions in the frequency domain.}

From a practical point of view, the signals $\boldsymbol{q}_{\phi}(t)$ we are interested in must have a finite duration of emission. However this is incompatible with the expression (17) of $\boldsymbol{q}_{\phi}(t)$. Indeed, from (5), it is clear that the Fourier transform $\hat{\boldsymbol{q}}_{\phi}$ of $\boldsymbol{q}_{\phi}$ is given by

$$
\hat{\boldsymbol{q}}_{\phi}(\omega)= \begin{cases}\chi(\omega) \mathrm{e}^{\mathrm{i} \phi(\omega)} \hat{\boldsymbol{w}}(\omega) & \text { if } \omega \in\left[\omega_{1}, \omega_{2}\right] \\ 0 & \text { if } \omega \in \mathbb{R}^{+} \backslash\left[\omega_{1}, \omega_{2}\right] \\ \overline{\hat{\boldsymbol{q}}_{\phi}(-\omega)} & \text { if } \omega \in \mathbb{R}^{-}\end{cases}
$$

In particular $\hat{\boldsymbol{q}}_{\phi}$ has a compact support in the frequency domain, so $\boldsymbol{q}_{\phi}$ cannot be compactly supported in the time domain since it is an entire function of $t$. However $\boldsymbol{q}_{\phi}(t)$ may be close to 0 outside a bounded interval provided that $\hat{\boldsymbol{q}}_{\phi}(\omega)$ is regular enough (recall that the Riemann-Lebesgue theorem tells us that if $t^{n} \boldsymbol{q}_{\phi}(t) \in L^{1}(\mathbb{R})$, then $\left.\hat{\boldsymbol{q}}_{\phi}(\omega) \in C^{n}(\mathbb{R})\right)$. In such a case, (17) can be considered as an approximation of a compactly supported signal.

It is then natural to assume at least that $\hat{\boldsymbol{q}}_{\phi}(\omega) \in C^{0}(\mathbb{R})$. This means on the one hand that $\chi$ and $\phi$ both belong to $C^{0}\left(\left[\omega_{1}, \omega_{2}\right]\right)$ and $\chi\left(\omega_{1}\right)=\chi\left(\omega_{2}\right)=0$, on the other hand that $\hat{\boldsymbol{w}}(\omega)$ depends also continuously on $\omega \in\left[\omega_{1}, \omega_{2}\right]$. This assumption is not restrictive. Indeed we can assume without loss of generality that our family of singular elements is continuous in the sense that $\lambda(\omega), \hat{\boldsymbol{v}}(\omega)$ and $\hat{\boldsymbol{w}}(\omega)$ are continuous functions of $\omega$. One can always find such a family. This follows from classical arguments of perturbation theory [14] using the fact that $\widehat{\mathbb{S}}_{\omega}$ is a continuous (even $C^{\infty}$ ) function of $\omega$ (which derives from the regularity of the time-harmonic Green's function $\hat{G}(\cdot, \cdot, \omega))$.

\section{A particular singular value decomposition.}

The phase choice we propose is related to the symmetry of the scattering operator (see remark 1). Indeed, this property implies that there exists a particular SVD, called the symmetric singular value decomposition (SSVD) or Takagi's factorization [13], which writes as

$$
\widehat{\mathbb{S}}_{\omega}=\overline{\mathbb{U}}_{\omega} \widehat{\mathbb{D}}_{\omega} \widehat{\mathbb{U}}_{\omega}^{*}
$$

where $\widehat{\mathbb{D}}_{\omega}$ is the diagonal matrix composed of the singular values and $\widehat{\mathbb{U}}_{\omega}$ is unitary. This decomposition is a particular version of $(14)$ with $\widehat{\mathbb{W}}_{\omega}=\widehat{\widehat{\mathbb{V}}}_{\omega}=\widehat{\mathbb{U}}_{\omega}$, i.e., the right and left singular vectors are conjugate of each other. From a practical point of view, the way to obtain the SSVD from a given SVD is straightforward, especially for simple singular values. Indeed, as $\widehat{\mathbb{S}}_{\omega}$ is symmetric, conjugating (16) yields

$$
\widehat{\mathbb{S}}_{\omega}^{*} \overline{\hat{\boldsymbol{w}}(\omega)}=\lambda(\omega) \overline{\hat{\boldsymbol{v}}(\omega)} \text { and } \quad \widehat{\mathbb{S}}_{\omega} \overline{\hat{\boldsymbol{v}}(\omega)}=\lambda(\omega) \overline{\hat{\boldsymbol{w}}(\omega)}
$$

This shows that if $(\hat{\boldsymbol{v}}(\omega), \hat{\boldsymbol{w}}(\omega))$ is a pair of left and right singular vectors, then so is $(\overline{\hat{\boldsymbol{w}}(\omega)}, \overline{\boldsymbol{\boldsymbol { v }}(\omega)})$. For a simple singular value $\lambda(\omega)$, we deduce from remark 2 that there exists $\theta(\omega) \in \mathbb{R}$ such that

$$
(\overline{\hat{\boldsymbol{w}}(\omega)}, \overline{\hat{\boldsymbol{v}}(\omega)})=\mathrm{e}^{\mathrm{i} \theta(\omega)}(\hat{\boldsymbol{v}}(\omega), \hat{\boldsymbol{w}}(\omega)) .
$$


As a consequence,

$$
\widehat{\mathbb{S}}_{\omega} \hat{\boldsymbol{w}}(\omega)=\mathrm{e}^{-\mathrm{i} \theta(\omega)} \lambda(\omega) \overline{\hat{\boldsymbol{w}}(\omega)} .
$$

This relation tells us how to change the phase of our right singular vector in order to obtain a singular vector of the SSVD (19). Indeed by setting

$$
\hat{\boldsymbol{w}}_{\mathrm{s}}(\omega):=\mathrm{e}^{\mathrm{i} \theta(\omega) / 2} \hat{\boldsymbol{w}}(\omega),
$$

we see that

$$
\widehat{\mathbb{S}}_{\omega} \hat{\boldsymbol{w}}_{\mathrm{s}}(\omega)=\lambda(\omega) \overline{\hat{\boldsymbol{w}}_{\mathbf{s}}(\omega)},
$$

which means that $\hat{\boldsymbol{w}}_{\mathrm{s}}(\omega)$ is a singular vector of the SSVD. Note that contrary to the singular vectors of a SVD (see remark 2$), \hat{\boldsymbol{w}}_{\mathrm{s}}(\omega)$ is defined uniquely up to a multiplication by -1 .

In the sequel our aim is to show that if we choose this particular singular vector $\hat{\boldsymbol{w}}_{\mathrm{s}}(\omega)$ instead of an arbitrary one $\hat{\boldsymbol{w}}(\omega)$ in the definition (17) of $\boldsymbol{q}_{\phi}(t)$, then the optimal choice for $\phi$ is either $\phi \equiv 0$ or $\phi \equiv \pi / 2$. We begin in the next section by proving that both phase functions lead to the minimum of a function which describes a time reversal process. But do they yield an optimal focusing? A ideal way to give a mathematical answer to this question would be to prove that these constant phase functions lead to the minimum of a function which would quantify the focus quality. Unfortunately, we were not able to find such a function. The only mathematical answer we can give is based on the connection between these constant phase functions and the time reversal experiment mentioned in the introduction. It is the subject of $\S 3.3$. We finally present in $\S 3.4$ some numerical results which confirm the space-time focusing effect.

\subsection{Optimality of a time reversal gap functional}

Hereafter we denote by $\mathbb{J}$ the time reversal transformation with origin $t=0$ : for every real-valued function $f=f(t)$, function $\mathbb{J} f$ is simply given by $\mathbb{J} f(t):=f(-t)$. Using the Fourier transform, it is readily seen that $\mathcal{F} \mathbb{J} f(\omega)=\overline{\mathcal{F} f(\omega)}$, or equivalently

$$
\mathbb{J}=\mathcal{F}^{-1} \widehat{\mathbb{J}} \mathcal{F} \quad \text { where } \quad \widehat{\mathbb{J}} \hat{f}:=\overline{\hat{f}} .
$$

This is nothing but the well-known fact that time reversal becomes complex conjugation in the frequency domain.

Our idea of using the SSVD originates from a heuristic argument which can be explained as follows. Suppose that the array emits several short pulse waves, respectively at times $t_{j}$ for $j=1, \ldots, J$, that propagate along different paths leading to the aimed scatterer. Let $T_{j}$ denote the travel time related to the $j$-th path. After the interaction of these incident waves with the scatterer, suppose that the associated scattered wave propagate back to the array along the same respective paths. Thus they will reach the array respectively at times $t_{j}^{\prime}:=t_{j}+2 T_{j}$ for $j=1, \ldots, J$. Obviously, the incident pulse waves will arrive at the same time $t_{*}$ on the scatterer only if $t_{j}+T_{j}=t_{*}$ for all $j$. In this case, the times of emission and reception become symmetric with respect to $t_{*}$ in the sense that $t_{j}^{\prime}-t_{*}=t_{*}-t_{j}$ for all $j$. More precisely, up to a scaling factor related to the reflectivity of the scatterer, the input and the output are time-reversed with respect to the focus time $t_{*}$.

This very simple model prompted us to search for phase functions such that this time reversal property could be nearly satisfied. This is the subject of Theorem 4 below which leads us to the SSVD in the case where the aimed focus time is $t_{*}=0$. Actually the connection between this time reversal property and the SSVD can be seen directly in the characterization (20) of the singular vectors of the SSVD, which can be written $\widehat{\mathbb{S}}_{\omega} \hat{\boldsymbol{w}}_{\mathrm{s}}(\omega)=\lambda(\omega) \widehat{\mathbb{J}} \hat{\boldsymbol{w}}_{\mathrm{s}}(\omega)$. This relation means that when the input signal sent to the transducers is $\hat{\boldsymbol{w}}_{\mathrm{s}}(\omega)$, then the measure of the scattered field is, up to a positive real factor $\lambda(\omega)$, the time reversed input signal. As relation (20) holds for all frequencies $\omega \in\left[\omega_{1}, \omega_{2}\right]$, all time-harmonic signals are time-reversed with respect to the same origin $t=0$. To a certain extent, this means that working with singular vectors of the SSVD amounts to synchronizing the time-harmonic emitted waves at time $t=0$. In the above comments, one can replace $\hat{\boldsymbol{w}}_{\mathrm{s}}(\omega)$ by i $\hat{\boldsymbol{w}}_{\mathrm{s}}(\omega)$. The only change is that the positive real factors become negative. These are the two possible choices which yield a synchronization at $t=0$. 
Theorem 4. Suppose that $\left\{\left(\lambda(\omega), \hat{\boldsymbol{w}}_{\mathrm{S}}(\omega)\right) \in \mathbb{R}^{+} \times \mathbb{C}^{N} ; \omega \in\left[\omega_{1}, \omega_{2}\right]\right\}$ is a continuous family of singular elements of the SSVD of $\widehat{\mathbb{S}}_{\omega}$. Let $\chi \in C^{0}\left(\left[\omega_{1}, \omega_{2}\right]\right)$ chosen such that $\chi\left(\omega_{1}\right)=\chi\left(\omega_{2}\right)=0$. For all $(\mu, \phi) \in$ $\mathbb{R} \times C^{0}\left(\left[\omega_{1}, \omega_{2}\right]\right)$, define

$$
\mathcal{G}(\mu, \phi):=\left\|(\mathbb{S}-\mu \mathbb{J}) \boldsymbol{q}_{\phi}\right\|_{\mathbb{R}_{t} ; \mathbb{C}^{N}}^{2}:=\int_{\mathbb{R}}\left\|(\mathbb{S}-\mu \mathbb{J}) \boldsymbol{q}_{\phi}(t)\right\|_{\mathbb{C}^{N}}^{2} \mathrm{~d} t
$$

where $\|\cdot\|_{\mathbb{C}^{N}}$ denotes the Euclidean norm in $\mathbb{C}^{N}$ and

$$
\boldsymbol{q}_{\phi}(t):=\frac{1}{\pi} \operatorname{Re} \int_{\omega_{1}}^{\omega_{2}} \chi(\omega) \mathrm{e}^{\mathrm{i} \phi(\omega)} \hat{\boldsymbol{w}}_{\mathrm{s}}(\omega) \mathrm{e}^{-\mathrm{i} \omega t} \mathrm{~d} \omega .
$$

Then the minimum of $\mathcal{G}(\mu, \phi)$ is reached at $\left(\mu_{+}, \phi_{+}\right)$and $\left(\mu_{-}, \phi_{-}\right)$where

$$
\mu_{ \pm}:= \pm \int_{\omega_{1}}^{\omega_{2}} \lambda(\omega) \chi(\omega)^{2} \mathrm{~d} \omega\left(\int_{\omega_{1}}^{\omega_{2}} \chi(\omega)^{2} \mathrm{~d} \omega\right)^{-1}, \quad \phi_{+} \equiv 0 \quad \text { and } \quad \phi_{-} \equiv \frac{\pi}{2} .
$$

Proof. Thanks to (8) and (21), Parseval's identity yields

$$
\mathcal{G}(\mu, \phi)=\frac{1}{2 \pi}\left\|(\widehat{\mathbb{S}}-\mu \widehat{\mathbb{J}}) \hat{\boldsymbol{q}}_{\phi}\right\|_{\mathbb{R}_{\omega} ; \mathbb{C}^{N}}^{2}=\frac{1}{2 \pi}\left(a_{\phi} \mu^{2}-2 b_{\phi} \mu+c_{\phi}\right),
$$

where $\hat{\boldsymbol{q}}_{\phi}:=\mathcal{F} \boldsymbol{q}_{\phi}$ and

$$
a_{\phi}:=\left\|\hat{\boldsymbol{q}}_{\phi}\right\|_{\mathbb{R}_{\omega} ; \mathbb{C}^{N}}^{2}, \quad b_{\phi}:=\operatorname{Re}\left(\widehat{\mathbb{S}} \hat{\boldsymbol{q}}_{\phi}, \widehat{\mathbb{J}} \hat{\boldsymbol{q}}_{\phi}\right)_{\mathbb{R}_{\omega} ; \mathbb{C}^{N}} \text { and } c_{\phi}:=\left\|\widehat{\mathbb{S}} \hat{\boldsymbol{q}}_{\phi}\right\|_{\mathbb{R}_{\omega} ; \mathbb{C}^{N}}^{2}
$$

Note that the expression of $a_{\phi}$ follows from the fact that $\widehat{\mathbb{J}}$ is an isometry. From (18) and (20), we deduce

$$
\begin{aligned}
a_{\phi} & =2 \int_{\omega_{1}}^{\omega_{2}} \chi(\omega)^{2} \mathrm{~d} \omega \\
b_{\phi} & =2 \int_{\omega_{1}}^{\omega_{2}} \lambda(\omega) \chi(\omega)^{2} \cos (2 \phi(\omega)) \mathrm{d} \omega \text { and } \\
c_{\phi} & =2 \int_{\omega_{1}}^{\omega_{2}} \lambda(\omega)^{2} \chi(\omega)^{2} \mathrm{~d} \omega .
\end{aligned}
$$

For a fixed $\phi$, the minimum of the second-order polynomial in the right-hand side of (22) is reached at $\mu_{\phi}:=b_{\phi} / a_{\phi}$. Moreover

$$
\mathcal{G}\left(\mu_{\phi}, \phi\right)=\frac{1}{2 \pi}\left(c_{\phi}-\frac{b_{\phi}^{2}}{a_{\phi}}\right)
$$

As $a_{\phi}$ and $c_{\phi}$ do not actually depend on $\phi$, the minimum of this function is reached for the maximum of $b_{\phi}^{2}$, that is, when $\cos (2 \phi(\omega))= \pm 1$. Hence $\phi(\omega)=k \pi / 2$ for some integer $k$. And of course it is sufficient to consider both cases $k=0$ and $k=1$ since the other cases correspond to the same signals $\boldsymbol{q}_{\phi}(t)$ or their opposites.

Function $\mathcal{G}(\mu, \phi)$ is a time reversal gap functional since it quantifies the gap between the measure of the scattered field and the time-reversed input signal rescaled by a real factor. If $\lambda(\omega)$ was constant in the whole frequency band $\left[\omega_{1}, \omega_{2}\right]$, say $\lambda(\omega)=\lambda_{\mathrm{c}}$, we would have $\mu_{ \pm}= \pm \lambda_{\mathrm{c}}$ and $\mathcal{G}\left(\mu_{ \pm}, \phi_{ \pm}\right)=0$. Hence $\boldsymbol{q}_{\phi_{ \pm}}(t)$ would be an eigenvector of the operator $\mathbb{J} \mathbb{S}$ associated with the eigenvalue $\pm \lambda_{c}$. In this case, the TR property satisfied in the frequency domain holds true in the time domain: the measure of the time-dependent scattered field is, up to a real factor $\pm \lambda_{\mathrm{c}}$, the time-reversed input signal. Theorem 4 tells us that in the general case, the phase functions $\phi_{+} \equiv 0$ and $\phi_{-} \equiv \pi / 2$ bring us as close as possible to this situation: $\boldsymbol{q}_{\phi_{ \pm}}(t)$ is close to some kind of eigenvector of $\mathbb{J} \mathbb{S}$. But of course, this is not really an eigenvector since the time reversal gap $\varepsilon:=\mathcal{G}\left(\mu_{ \pm}, \phi_{ \pm}\right)$does not vanish in general. It can be interpreted by means of the notion of pseudospectra [23]: $\mu_{ \pm}$belongs to the $\varepsilon$-pseudospectrum of $\mathbb{J} \mathbb{S}$ and $\boldsymbol{q}_{\phi_{ \pm}}(t)$ is a corresponding $\varepsilon$-pseudoeigenvector. 


\subsection{Space-time focusing: from active sources to passive scatterers}

In the previous section, the link between Theorem 4 and space-time focusing is only based on a heuristic argument. The purpose of this section is to reinforce this link by a mathematical argument: we show that under certain conditions, the optimal signals of Theorem 4 yield the same space-time refocusing as the time reversal experiment for active sources which is mentioned in the introduction.

\section{Mathematical interpretation of the time reversal experiment.}

Suppose that at some point $y \in \mathbb{R}^{d}$, an active point-like source emits a short pulse defined by

$$
v_{\text {act }}^{(\ell)}(x, t):=\frac{1}{\pi} \operatorname{Re} \int_{\omega_{1}}^{\omega_{2}} \chi_{\text {act }}(\omega)(-\mathrm{i} \omega)^{\ell} \hat{G}(x, y, \omega) \mathrm{e}^{-\mathrm{i} \omega t} \mathrm{~d} \omega
$$

where $\ell \in \mathbb{N}$ and $\chi_{\text {act }}$ is a cutoff function chosen as in Theorem 4 . This wave can be seen as a spectral truncation of a time-derivative of the time-dependent Green's function since $v_{\text {act }}^{(\ell)}(x, t)=\left(\partial^{\ell} / \partial t^{\ell}\right) G(x, y, t)$ if $\left(\omega_{1}, \omega_{2}\right)=(0,+\infty)$ and $\chi_{\text {act }} \equiv 1$ (see (5)). Except in this latter situation, $v_{\text {act }}^{(\ell)}$ is not causal but it can be considered as an approximation of a causal function for a large enough frequency band $\left(\omega_{1}, \omega_{2}\right)$. The measure of $v_{\text {act }}^{(\ell)}$ by the array is then given by

$$
\begin{aligned}
\boldsymbol{q}^{(\ell)}(t) & =\frac{1}{\pi} \operatorname{Re} \int_{\omega_{1}}^{\omega_{2}} \chi_{\text {act }}(\omega)(-\mathrm{i} \omega)^{\ell} \hat{\boldsymbol{g}}(\omega) \mathrm{e}^{-\mathrm{i} \omega t} \mathrm{~d} \omega \text { where } \\
\hat{\boldsymbol{g}}(\omega) & :=\left(\hat{G}\left(x_{1}, y, \omega\right), \ldots, \hat{G}\left(x_{N}, y, \omega\right)\right)^{\top} .
\end{aligned}
$$

The idea of the TR experiment consists in using the time-reversed measure

$$
\mathbb{J} \boldsymbol{q}^{(\ell)}(t)=\boldsymbol{q}^{(\ell)}(-t)=\frac{1}{\pi} \operatorname{Re} \int_{\omega_{1}}^{\omega_{2}} \chi_{\mathrm{act}}(\omega)(\mathrm{i} \omega)^{\ell} \overline{\hat{\boldsymbol{g}}(\omega)} \mathrm{e}^{-\mathrm{i} \omega t} \mathrm{~d} \omega
$$

as input signals to emit a new wave

$$
v_{\text {back }}^{(\ell)}(x, t):=\frac{1}{\pi} \operatorname{Re} \int_{\omega_{1}}^{\omega_{2}} \chi_{\text {act }}(\omega)(\mathrm{i} \omega)^{\ell}\left(\sum_{n=1}^{N} \overline{\hat{G}\left(x_{n}, y, \omega\right)} \hat{G}\left(x, x_{n}, \omega\right)\right) \mathrm{e}^{-\mathrm{i} \omega t} \mathrm{~d} \omega .
$$

The fact that this wave propagates back to the original source can be understood easily in the case of a full aperture spherical array located far enough from the inhomogeneities of the medium. Assume that the family of points $x_{n}$ is distributed regularly on the surface $S_{R}:=\left\{z \in \mathbb{R}^{d} ;\|z\|=R\right\}$. Hence, in the above formula, the sum can be interpreted as an approximation of an integral on $S_{R}$, that is,

$$
\sum_{n=1}^{N} \overline{\hat{G}\left(x_{n}, y, \omega\right)} \hat{G}\left(x, x_{n}, \omega\right) \approx C \int_{S_{R}} \overline{\hat{G}(z, y, \omega)} \hat{G}(x, z, \omega) \mathrm{d} S(z)
$$

where $C>0$ depends on $N$ and the space dimension $d$. The asymptotic behaviour of the latter integral for large $\mathrm{R}$ follows from the Helmholtz-Kirchhoff formula [11]:

$$
\int_{S_{R}} \overline{\hat{G}(z, y, \omega)} \hat{G}(x, z, \omega) \mathrm{d} S(z) \stackrel{R \rightarrow+\infty}{\approx} \frac{\hat{G}(x, y, \omega)-\overline{\hat{G}(x, y, \omega)}}{2 \mathrm{i} \omega} \text { for }\|x\| \ll R
$$

As a consequence,

$$
v_{\text {back }}^{(\ell)}(x, t) \approx \frac{C}{2 \pi} \operatorname{Re} \int_{\omega_{1}}^{\omega_{2}} \chi_{\text {act }}(\omega)(\mathrm{i} \omega)^{\ell-1}(\hat{G}(x, y, \omega)-\overline{\hat{G}(x, y, \omega)}) \mathrm{e}^{-\mathrm{i} \omega t} \mathrm{~d} \omega .
$$

Going back to the expression $(23)$ of $v_{\text {act }}^{(\ell)}$, we conclude that

$$
v_{\mathrm{back}}^{(\ell)}(x, t) \approx \frac{C}{2}\left((-1)^{\ell-1} v_{\mathrm{act}}^{(\ell-1)}(x, t)-v_{\mathrm{act}}^{(\ell-1)}(x,-t)\right) .
$$


In this expression, $v_{\text {act }}^{(\ell-1)}(x, t)$ approximates a time-derivative of the Green's function, which is causal, whereas the other term $v_{\text {act }}^{(\ell-1)}(x,-t)$ represents the time-reversed of this function and then appears as an approximation of an anti-causal function. As a consequence,

$$
v_{\text {back }}^{(\ell)}(x, t) \approx \begin{cases}-\frac{C}{2} v_{\text {act }}^{(\ell-1)}(x,-t) & \text { if } t<0, \\ (-1)^{\ell-1} \frac{C}{2} v_{\text {act }}^{(\ell-1)}(x, t) & \text { if } t>0 .\end{cases}
$$

This means that for $t<0, v_{\text {back }}^{(\ell)}$ behaves like a converging wave which propagates towards the initial source $y$ and refocuses at this point at time $t=0$, whereas for $t>0, v_{\text {back }}^{(\ell)}$ diverges from $y$. This gives us a mathematical interpretation of the TR experiment.

\section{Back to passive scatterers.}

Let us now come back to our problem and suppose for simplicity that there is only one scatterer located at $y$. In this case, the expression (13) of the time-harmonic scattering operator simplifies as

$$
\widehat{\mathbb{S}}_{\omega}=\sigma(\omega) \hat{\boldsymbol{g}}(\omega) \hat{\boldsymbol{g}}(\omega)^{\top}
$$

where $\sigma(\omega)$ is the reflectivity coefficient of the scatterer (see (9)) and $\hat{\boldsymbol{g}}(\omega)$ is defined in (24). This shows in particular that the rank of $\widehat{\mathbb{S}}_{\omega}$ is 1 . Moreover,

$$
\widehat{\mathbb{S}}_{\omega} \overline{\mathrm{e}^{\mathrm{i} \arg (\sigma(\omega)) / 2} \hat{\boldsymbol{g}}(\omega)}=|\sigma(\omega)|\|\hat{\boldsymbol{g}}(\omega)\|_{\mathbb{C}^{N}}^{2}\left(\mathrm{e}^{\mathrm{i} \arg (\sigma(\omega)) / 2} \hat{\boldsymbol{g}}(\omega)\right)
$$

which means that

$$
\lambda(\omega):=|\sigma(\omega)|\|\hat{\boldsymbol{g}}(\omega)\|_{\mathbb{C}^{N}}^{2} \quad \text { and } \quad \hat{\boldsymbol{w}}_{\mathrm{s}}(\omega):=\frac{\mathrm{e}^{-\mathrm{i} \arg (\sigma(\omega)) / 2} \overline{\hat{\boldsymbol{g}}(\omega)}}{\|\hat{\boldsymbol{g}}(\omega)\|_{\mathbb{C}^{N}}}
$$

are singular elements of the SSVD of $\widehat{\mathbb{S}}_{\omega}$. From $(9)$, we infer that $\arg (\sigma(\omega))$ either vanishes (if $d=3$ ) or is small (if $d=2$ : the asymptotic of $H_{0}^{(1)}$ for small arguments shows that $\arg (\sigma(\omega))=O\left((\ln \varepsilon)^{-1}\right)$ ). Hence $\hat{\boldsymbol{w}}_{\mathrm{s}}(\omega) \approx \overline{\hat{\boldsymbol{g}}(\omega)} /\|\hat{\boldsymbol{g}}(\omega)\|_{\mathbb{C}^{N}}$. Thus the respective behaviours of the optimal signals of Theorem 4 are given by

$$
\boldsymbol{q}_{\phi_{ \pm}}(t) \approx \frac{1}{\pi} \operatorname{Re} \int_{\omega_{1}}^{\omega_{2}} \chi(\omega) \mathrm{e}^{\mathrm{i} \phi_{ \pm}} \frac{\overline{\hat{\boldsymbol{g}}(\omega)}}{\|\hat{\boldsymbol{g}}(\omega)\|_{\mathbb{C}^{N}}} \mathrm{e}^{-\mathrm{i} \omega t} \mathrm{~d} \omega
$$

where we recall that $\phi_{+} \equiv 0$ and $\phi_{-} \equiv \pi / 2$. Comparing the latter formula with $(25)$, we see that if both cutoff functions $\chi$ and $\chi$ act satisfy

$$
\chi(\omega)=\chi_{\text {act }}(\omega) \omega^{\ell}\|\hat{\boldsymbol{g}}(\omega)\|_{\mathbb{C}^{N}},
$$

then, up to a change of sign, $\boldsymbol{q}_{\phi_{+}}(t)$ (respectively, $\boldsymbol{q}_{\phi_{-}}(t)$ ) behaves like $\boldsymbol{q}^{(\ell)}(-t)$ for even $\ell$ (respectively, odd $\ell$ ). We conclude that the wave emitted by an optimal signal of Theorem 4 is similar to the backpropagating wave produced by the TR experiment when the initial wave is some spectral truncation of a time-derivative of the Green's function. In short, our method for passive scatterers yields some kind of optimal space-time focusing. This is justified above for one single scatterer. Using similar arguments as in [12], it is easy to see that this holds true for distant enough scatterers. The next section illustrates these results and also shows that the focus quality decreases if the scatterers become close.

\subsection{Numerical results}

We finally present some numerical experiments which confirm the space-time focusing effect related to the optimal signals of Theorem 4 . We consider the same geometrical configuration as in section 2.2. We use the family of singular vectors of the SSVD of $\widehat{\mathbb{S}}_{\omega}$ associated with the dominant eigenvalue (which 

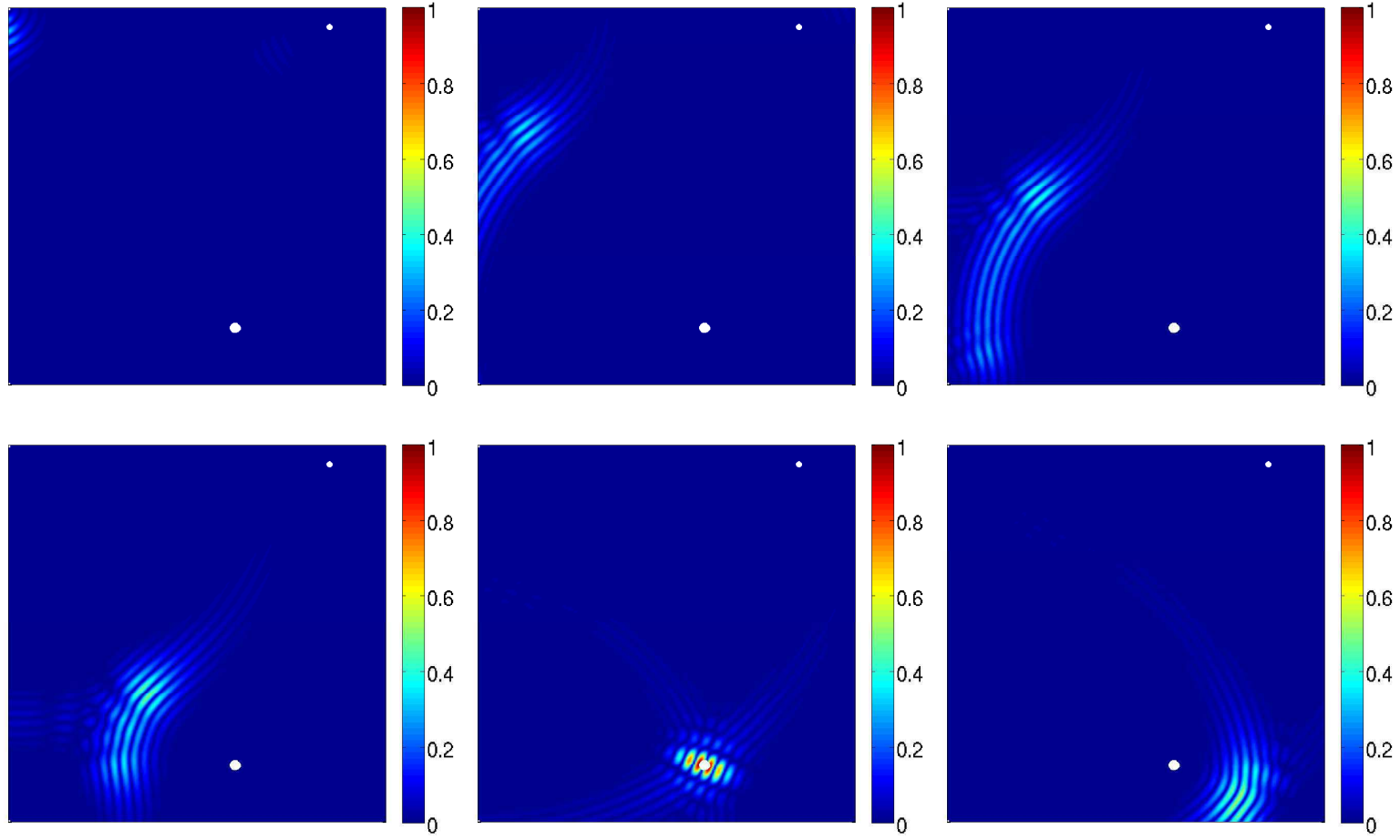

Figure 4: In the case of a homogeneous medium, modulus of the field generated by the optimal signal $\boldsymbol{q}_{\phi_{+}}(t)$ of Theorem 4 at times $t=-2.0,-1.4,-1.0,-0.6,0.0$ and 0.4 (the field is rescaled so that its modulus belongs to $[0,1])$.
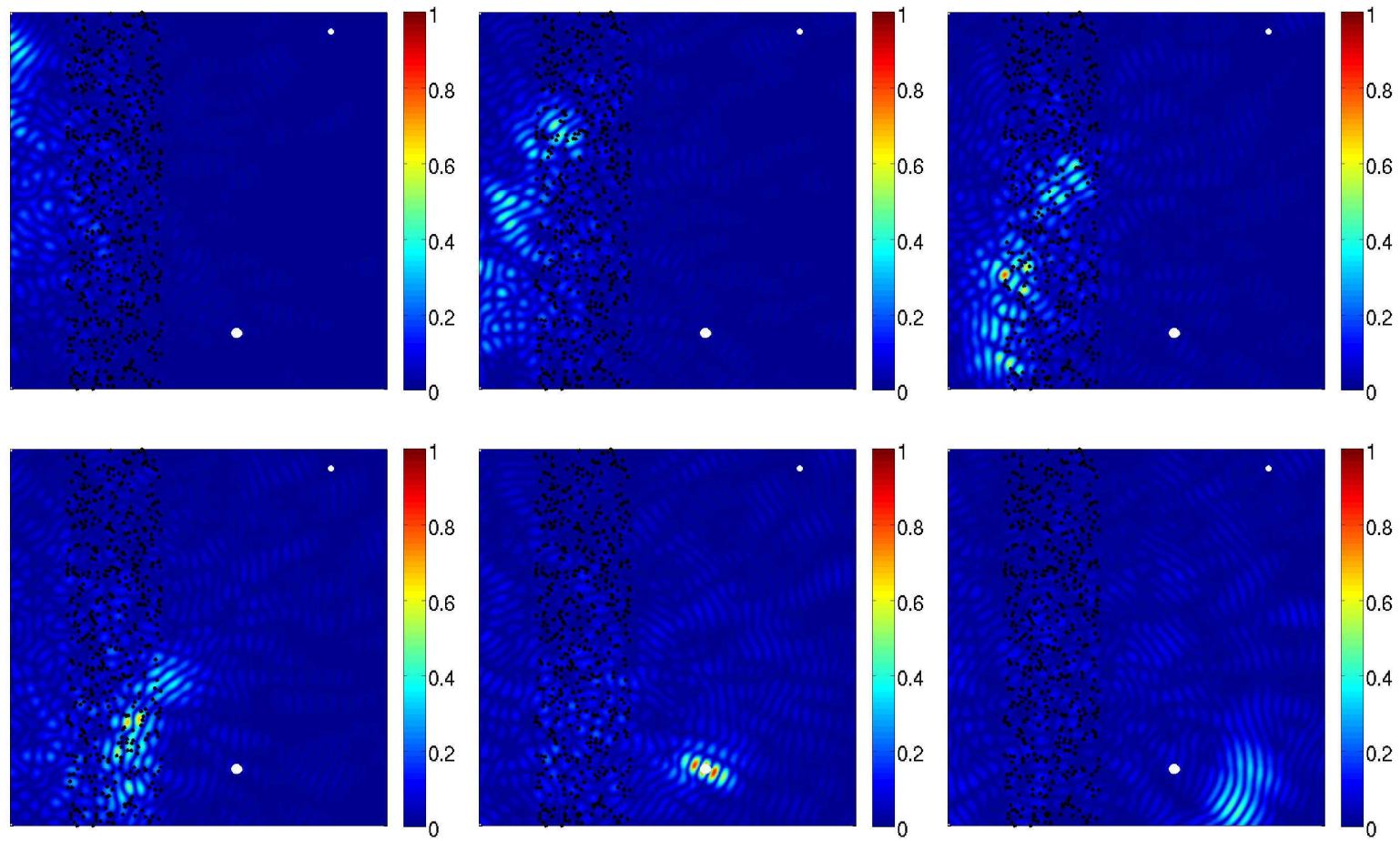

Figure 5: Same as Figure 4 in the case of a scattering medium. 
focus in space on the biggest scatterer, located at $(1.2,0.3))$. The cutoff function we have chosen is a truncated Gaussian function given by

$$
\chi(\omega)=\mathbf{1}_{\left[\omega_{1}, \omega_{2}\right]}(\omega) \exp \left(\frac{\left(\omega-\omega_{0}\right)^{2}}{2 \gamma^{2}}\right)
$$

where $\left[\omega_{1}, \omega_{2}\right]=[20,100], \omega_{0}=60$ and $\gamma=10$. Note that strictly speaking, this function is not continuous at $\omega_{1}$ and $\omega_{2}$ as assumed in Theorem 4 . However the Gaussian function is very small at these points (about $\left.3 \times 10^{-4}\right)$.

Figure 4 shows the evolution of the wave generated by the optimal signal $\boldsymbol{q}_{\phi_{+}}(t)$ of Theorem 4 in the case of a homogeneous medium. The space-time focusing appears very clearly: the array seems to emit an angular portion of a radial wave which propagates towards the target and concentrates near it at time $t=0$. In this situation, the structure of the signal is very simple: Figure 6 (left) shows that all the transducers emit approximatively the same signal starting at different times which only depend on the distances between the transducers and the target.

Figure 5 illustrates the influence of the same scattering medium as in section 2.2, i.e., 500 point-like scatterers randomly displayed in the rectangle $[0.3,0.8] \times[0,2]$. Again space-time focusing is confirmed. The focal spot observed at time $t=0$ is even slightly smaller than in the homogeneous case, which is the well-known super-resolution effect of a scattering medium (see, e.g., $[3,5])$. On the other hand, the structure of the wave is strongly perturbed. Resonance phenomena are observed inside the scattering medium, which slow down the propagation of the wave. In order to compensate these effects, a part of the wave is emitted earlier so that the various components meet at the same time $t=0$ near the target. This is shown in Figure 6 (right) where we recognize a main front whose shape is similar to the homogeneous case, but which is now preceded by an unstructured contribution.

The above comments remain valid if we use the optimal signal $\boldsymbol{q}_{\phi_{-}}(t)$ of Theorem 4 instead of $\boldsymbol{q}_{\phi_{+}}(t)$. Actually the differences between both cases are not so easy to detect. Figure 7 shows a zoom on the focal spots observed at $t=0$ in the case of a homogeneous medium. We see that both focal spots are about the same size. They seem to be shifted by a quarter wavelength, which is consistent with the fact that in the frequency domain, both signals are in phase quadrature.

In order to quantify the focus quality, we propose an energy criterion which simply consists in comparing the acoustic energy concentrated in a given vicinity of the target with the total energy emitted by the array of transducers. Let $u=u(x, t)$ denote the wave emitted in the reference medium by the array for some input signal $\boldsymbol{q}(t)$ (see (2)). For a given box $B \subset \mathbb{R}^{d}$ around our target, we define a focus quality function by

$$
\mathcal{Q}_{B}(t):=\frac{\mathcal{E}_{B}(t)}{\mathcal{E}_{\text {tot }}} \quad \text { where } \quad \mathcal{E}_{B}(t):=\frac{1}{2} \int_{B}\left(\frac{\partial u}{\partial t}(x, t)\right)^{2}+|\nabla u(x, t)|^{2} \mathrm{~d} x
$$

represents the local acoustic energy contained in $B$ at time $t$ (see (27)) and $\mathcal{E}_{\text {tot }}$ stands for the total energy emitted by the array. As our array consists of point-like transducers, the fact that $\mathcal{E}_{\text {tot }}$ is finite is not obvious. As a matter of fact, we show in the appendix that during the emission, this energy is infinite, but becomes finite as soon as the transducers become silent (provided that the input signals are regular enough). A suitable expression of $\mathcal{E}_{\text {tot }}$ is given in Proposition 5.

Figure 8 compares the focus quality functions computed for two signals: on the one hand, the optimal signal $\boldsymbol{q}_{\phi_{+}}(t)$ of Theorem 4 and on the other hand, the signal (25) of the TR experiment with $\ell=0$ and $\chi_{a c t}=\chi$. On the left, we consider the same configuration as in Figure 4, that is, the case of the homogeneous medium. We see that both curves coincide, which is consistent with the results of section 3.3. The maximum of $\mathcal{Q}_{B}(t)$ is reached at time $t=0$ with $44 \%$ of the total energy concentrated in the box represented in Figure 7. Note that in our model, the array emits the same wave on both sides, so $\mathcal{Q}_{B}(t)$ cannot exceed $50 \%$. The case of the scattering medium of Figure 5 is represented in the middle part. Both curves nearly coincide, which confirms again the results of section 3.3, but here the maximum of $\mathcal{Q}_{B}(t)$ is only $15 \%$, which shows that a great part of the energy is 'lost' in the scattering medium. Finally, in the right part, we consider again the scattering medium but the second obstacle is moved closer to the target. Of course, $\mathcal{Q}_{B}(t)$ is unchanged for the signal of the TR experiment since it does not depend on the second obstacle. On the other hand, the focus quality decreases for the optimal signal 

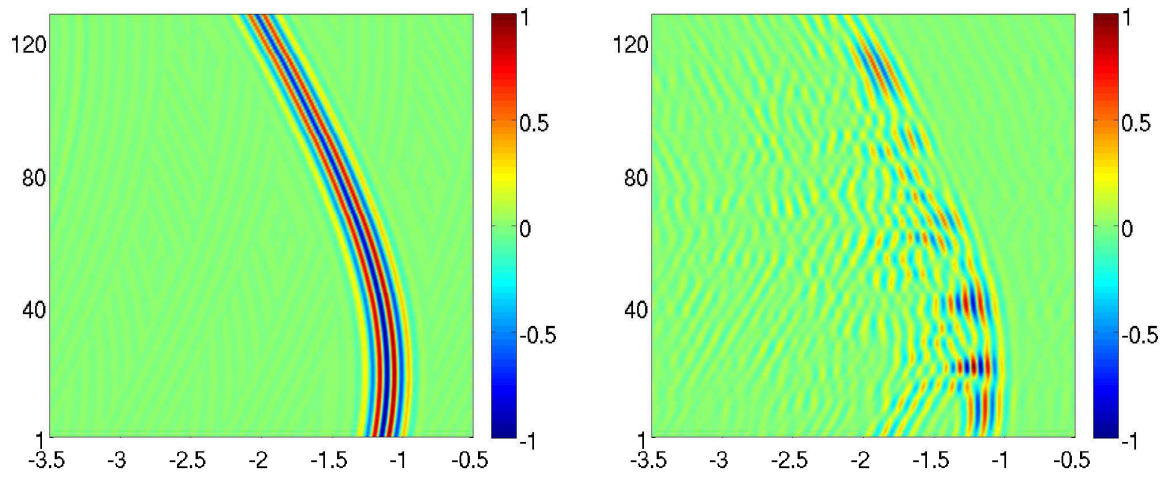

Figure 6: Representation of the optimal signal $\boldsymbol{q}_{\phi_{+}}(t)$ of Theorem 4 (rescaled in $[-1,+1]$ ) in the homogeneous medium (left) and in the presence of the scattering medium (right). The horizontal coordinate is time and the vertical one is the transducer's number.
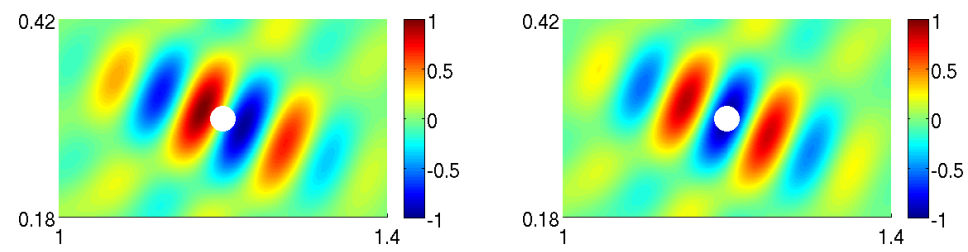

Figure 7: In the case of a homogeneous medium, zoom in the box $[1.0,1.4] \times[0.18,0.42]$ of the focal spots obtained at time $t=0$ with the optimal signals $\boldsymbol{q}_{\phi_{+}}(t)$ (left) and $\boldsymbol{q}_{\phi_{-}}(t)$ (right) of Theorem 4.
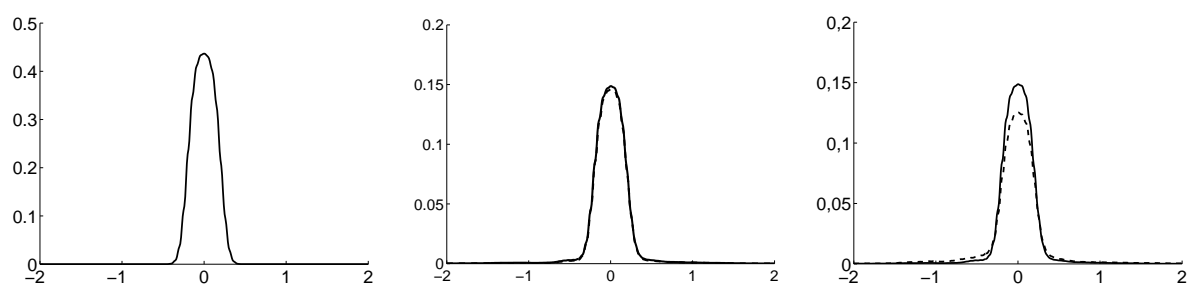

Figure 8: Representation of the focus quality function $\mathcal{Q}_{B}(t)$ for $t \in[-2,+2]$ and the box $B$ of Figure 7 in three configurations: the homogeneous medium of Figure 4 (left), the scattering medium of Figure 5 (middle) and the same scattering medium with the second scatterer moved at point $(0.5,1.6)$ (right). For each case, the dashed line corresponds to the optimal signal $\boldsymbol{q}_{\phi_{+}}(t)$ of Theorem 4 and the solid line, the signal (25) of the TR experiment with $\ell=0$ and $\chi_{a c t}=\chi$. 
$\boldsymbol{q}_{\phi_{+}}(t)$ (maximum at $12.5 \%$ ). This is a well-known effect of the DORT method, which maximizes the energy of the scattered wave and then leads to illuminate all obstacles when they are close to each other.

\section{Conclusion}

In the present paper, we have shown that the use of the symmetric singular value decomposition (SSVD) of the time-harmonic scattering operator allows us to synchronize its singular vectors in the frequency domain in order to achieve space-time focusing. We have developed several theoretical and numerical arguments which support this assertion. In particular, we have seen that for distant enough scatterers, the choice of the SSVD for unknown passive scatterers yields the same refocusing as time reversal for an active source, which can be considered as an optimal refocusing. Nevertheless two criticisms can be made.

On the one hand, from a practical point of view, the principle of DORT method is based on the measure of the scattered wave. As mentioned in the introduction, this means that one has to measure separately the incident and the perturbed waves (that is, in the absence and in the presence of the target) and compute their difference. In many situations, it is impossible to make this double measure. However when the medium is weakly inhomogeneous and when the array is located on one side of the medium (open aperture case), only a tiny part of the incident wave propagates back to the array so that one can consider that one measures directly the scattered field.

On the other hand, from a mathematical point of view, it is quite frustrating to be unable to relate the use of the SSVD to a mathematical criterion which should evaluate the quality of space-time focusing. The energy criterion proposed in section 3.4 seems very natural. But can we affirm that according to this criterion or another focusing criterion, the best signals are those of Theorem 4? The question remains open.

\section{Appendix: Energy emitted by point sources}

Let $\Omega$ be a domain of $\mathbb{R}^{d}$, bounded or not, with boundary $\Gamma$ and $u=u(x, t)$ a causal solution to the acoustic wave equation

$$
\frac{\partial^{2} u}{\partial t^{2}}-\Delta u=f \quad \text { in } \Omega \times \mathbb{R}
$$

for a given causal function $f=f(x, t)$. By multiplying this equation by $\partial u / \partial t$ and integrating over $\Omega$, we deduce classically from Green's formula the energy conservation law:

$$
\frac{\mathrm{d}}{\mathrm{d} t} \mathcal{E}_{\Omega}(t)=\mathcal{P}_{\Omega}(t)+\mathcal{F}_{\Gamma}(t)
$$

where $\mathcal{E}_{\Omega}(t)$ denotes the acoustic energy at time $t, \mathcal{P}_{\Omega}(t)$ is the power produced by the source $f$ and $\mathcal{F}_{\Gamma}(t)$ is the incoming energy flux across $\Gamma$, which are defined respectively by

$$
\begin{aligned}
\mathcal{E}_{\Omega}(t) & :=\frac{1}{2} \int_{\Omega}\left(\frac{\partial u}{\partial t}(x, t)\right)^{2}+|\nabla u(x, t)|^{2} \mathrm{~d} x, \\
\mathcal{P}_{\Omega}(t) & :=\int_{\Omega} f(x, t) \frac{\partial u}{\partial t}(x, t) \mathrm{d} x \text { and } \\
\mathcal{F}_{\Gamma}(t) & :=\int_{\Gamma} \frac{\partial u}{\partial \nu}(x, t) \frac{\partial u}{\partial t}(x, t) \mathrm{d} \gamma_{x},
\end{aligned}
$$

where $\nu$ is the unit normal on $\Gamma$ pointing outside $\Omega$. Formula (26) holds provided that $u$ is regular enough so that the definitions above make sense, for instance if $f \in L^{2}(\Omega \times \mathbb{R})$. The purpose of this appendix is to deal with the case where $f$ represents the excitation produced by point sources located at $x_{n}$ for $n=1, \ldots, N$, i.e.,

$$
f(x, t)=\sum_{n=1}^{N} q_{n}(t) \delta\left(x-x_{n}\right),
$$


where functions $q_{n}$ are assumed causal. In this case, definitions (27)-(29) are not adapted. Indeed, because of the singular behaviour of $u$ near the sources, the integrals on $\Omega$ diverge, which means that the usual acoustic energy is infinite for point sources. However we show below that this energy becomes finite as soon as the sources become silent. Our aim is to exhibit an explicit expression of this energy. Surprisingly, we did not succeed in finding one in the literature!

We consider only the cases of dimensions 2 and 3. Formula (2) provides us the field produced by point sources. Thanks to the expression (1) of the Green's function in a homogeneous medium, it can be written more explicitly as

$$
\begin{aligned}
u & =\sum_{n=1}^{N} u_{n} \text { where } \\
u_{n}(x, t) & := \begin{cases}\int_{\left|x-x_{n}\right|}^{\max \left\{t,\left|x-x_{n}\right|\right\}} \frac{q_{n}(t-\tau)}{2 \pi \sqrt{\tau^{2}-\left|x-x_{n}\right|^{2}}} \mathrm{~d} \tau & \text { if } d=2, \\
\frac{q_{n}\left(t-\left|x-x_{n}\right|\right)}{4 \pi\left|x-x_{n}\right|} & \text { if } d=3 .\end{cases}
\end{aligned}
$$

We see here that if $q_{n}$ is regular enough, then $u_{n}$ has a similar regularity outside a vicinity of $x_{n}$. Hence, outside a collection of small balls $B_{n}(\varepsilon):=\left\{x \in \mathbb{R}^{d} ;\left|x-x_{n}\right| \leq \varepsilon\right\}$, energy conservation (26) holds. We shall use an integrated version of this equation:

$$
\mathcal{E}_{\Omega(\varepsilon)}(t)=\int_{0}^{t} \mathcal{F}_{\Gamma(\varepsilon)}(s) \mathrm{d} s,
$$

where $\Omega(\varepsilon):=\mathbb{R}^{d} \backslash \cup_{n=1, N} B_{n}(\varepsilon)$ and $\Gamma(\varepsilon):=\partial \Omega(\varepsilon)=\cup_{n=1, N} \partial B_{n}(\varepsilon)$ (note that $\mathcal{P}_{\Omega(\varepsilon)}(t)=0$ since $f$ vanishes in $\Omega(\varepsilon)$ ). The following proposition tells us that we can pass to the limit $\varepsilon \rightarrow 0$ in this equation when the sources become silent.

Proposition 5. Suppose that all functions $q_{n}$ belong to $C^{2}(\mathbb{R})$ and have a compact support contained in $[0, T]$ for some $T>0$. Then for all $t>T$, the limit $\lim _{\varepsilon \rightarrow 0} \mathcal{E}_{\Omega(\varepsilon)}(t)$ exists and is independent of $t$. It is given by

$$
\begin{aligned}
& \mathcal{E}_{\mathrm{tot}}=\sum_{n=1}^{N} \sum_{m=1}^{N} \mathcal{E}_{n m} \text { where } \\
& \mathcal{E}_{n n}:= \begin{cases}\int_{0}^{T} \int_{\tau}^{T} q_{n}^{\prime}(s-\tau) q_{n}(s) \mathrm{d} s \frac{\mathrm{d} \tau}{2 \pi \tau} & \text { if } d=2, \\
\int_{0}^{T} \frac{q_{n}^{\prime}(s)^{2}}{4 \pi} \mathrm{d} s & \text { if } d=3,\end{cases} \\
& \mathcal{E}_{n m}:=\int_{0}^{T} q_{n}(s) \frac{\partial u_{m}}{\partial t}\left(x_{n}, s\right) \mathrm{d} s \quad \text { for } n \neq m .
\end{aligned}
$$

Remark 6. Using Parseval's identity, these expressions are easily converted to the frequency domain. We obtain

$$
\begin{aligned}
& \mathcal{E}_{n n}:= \begin{cases}\frac{-1}{2 \pi} \int_{0}^{T} \frac{1}{\tau} \frac{\mathrm{d}}{\mathrm{d} \tau}\left(\mathcal{F}^{-1}\left(\left|\hat{q}_{n}\right|^{2}\right)\right)(\tau) \mathrm{d} \tau & \text { if } d=2 \\
\frac{1}{4 \pi^{2}} \int_{0}^{+\infty}\left|\omega \hat{q}_{n}(\omega)\right|^{2} \mathrm{~d} \omega & \text { if } d=3\end{cases} \\
& \mathcal{E}_{n m}:=-\frac{1}{\pi} \operatorname{Re} \int_{0}^{+\infty} \mathrm{i} \omega \hat{G}\left(x_{m}, x_{n}, \omega\right) \hat{q}_{m}(\omega) \overline{\hat{q}_{n}(\omega)} \mathrm{d} \omega \text { for } n \neq m \text {. }
\end{aligned}
$$

In the above formulas, $\mathcal{E}_{n n}$ represents the energy produced by the source located at $x_{n}$ if it was alone, whereas the other terms $\mathcal{E}_{n m}$ for $n \neq m$ stand for the interactions between the sources. In order to prove Proposition 5, we need to know the behaviour of $u_{n}$ near $x_{n}$, which is summarized in the following lemma. 
Lemma 7. Suppose that $q_{n} \in C^{2}(\mathbb{R})$ is causal. Then $u_{n} \in C^{2}\left(\left(\mathbb{R}^{d} \backslash B_{n}(\varepsilon)\right) \times \mathbb{R}\right)$ for all $\varepsilon>0$. Moreover, when $\varepsilon$ tends to 0 , the following asymptotic expansions hold uniformly for $x \in \partial B_{n}(\varepsilon)$ and $t \in[0, T]$ for any given $T>0$ :

$$
\begin{gathered}
\frac{\partial u_{n}}{\partial t}(x, t)= \begin{cases}\frac{1}{2 \pi}\left(\int_{\varepsilon}^{\max \{t, \varepsilon\}} \frac{q_{n}^{\prime}(t-\tau)}{\tau^{2}} \sqrt{\tau^{2}-\varepsilon^{2}} \mathrm{~d} \tau+q_{n}^{\prime}(t)\right)+O(\varepsilon) & \text { if } d=2, \\
\frac{q_{n}^{\prime}(t)}{4 \pi \varepsilon}-\frac{q_{n}^{\prime \prime}(t)}{4 \pi}+o(1) & \text { if } d=3,\end{cases} \\
\frac{\partial u_{n}}{\partial \nu}(x, t)= \begin{cases}\frac{q_{n}(t)}{2 \pi \varepsilon}+O(1) & \text { if } d=2, \\
\frac{q_{n}(t)}{4 \pi \varepsilon^{2}}+O(1) & \text { if } d=3 .\end{cases}
\end{gathered}
$$

Moreover, if $d=2$, the integral involved in the expression of $\partial u_{n} / \partial t$ is $O(|\log \varepsilon|)$.

Proof. The case $d=3$ is easy. From (31), we see that $u_{n} \in C^{2}\left(\left(\mathbb{R}^{3} \backslash B_{n}(\varepsilon)\right) \times \mathbb{R}\right)$ and for $x \in \partial B_{n}(\varepsilon)$, we have

$$
\frac{\partial u_{n}}{\partial t}(x, t)=\frac{q_{n}^{\prime}(t-\varepsilon)}{4 \pi \varepsilon} \quad \text { and } \quad \frac{\partial u_{n}}{\partial \nu}(x, t)=\frac{q_{n}^{\prime}(t-\varepsilon)}{4 \pi \varepsilon}+\frac{q_{n}(t-\varepsilon)}{4 \pi \varepsilon^{2}} .
$$

Using a Taylor expansion of $q_{n}(t-\varepsilon)$ and $q_{n}^{\prime}(t-\varepsilon)$, the conclusion follows.

The case $d=2$ is more intricate. The $C^{2}$-regularity of $u_{n}$ is easily deduced from its expression (31), which shows in addition that for $x \in \partial B_{n}(\varepsilon)$,

$$
\frac{\partial u_{n}}{\partial t}(x, t)=\frac{1}{2 \pi} \int_{\varepsilon}^{t_{\varepsilon}} \frac{q_{n}^{\prime}(t-\tau)}{\rho_{\varepsilon}(\tau)} \mathrm{d} \tau
$$

where $\rho_{\varepsilon}(\tau):=\sqrt{\tau^{2}-\varepsilon^{2}}$ and $t_{\varepsilon}:=\max \{t, \varepsilon\}$. Noticing that $\rho_{\varepsilon}^{\prime}(\tau)=\tau / \rho_{\varepsilon}(\tau)$ and integrating by part, we deduce that

$$
\frac{\partial u_{n}}{\partial t}(x, t)=\frac{1}{2 \pi} \int_{\varepsilon}^{t_{\varepsilon}}\left(\frac{q_{n}^{\prime}(t-\tau)}{\tau^{2}}+\frac{q_{n}^{\prime \prime}(t-\tau)}{\tau}\right) \rho_{\varepsilon}(\tau) \mathrm{d} \tau .
$$

The first term in the integral yields the dominant contribution, which is $O(|\log \varepsilon|)$ because

$$
\left|\int_{\varepsilon}^{t_{\varepsilon}} \frac{q_{n}^{\prime}(t-\tau)}{\tau^{2}} \rho_{\varepsilon}(\tau) \mathrm{d} \tau\right| \leq \sup _{\tau \in[0, t]}\left|q_{n}^{\prime}(\tau)\right| \int_{\varepsilon}^{t_{\varepsilon}} \frac{\mathrm{d} \tau}{\tau},
$$

since $\rho_{\varepsilon}(\tau) \leq \tau$ for all $\tau \geq \varepsilon$. To evaluate the second one, rewrite it as

$$
\int_{\varepsilon}^{t_{\varepsilon}} \frac{q_{n}^{\prime \prime}(t-\tau)}{\tau} \rho_{\varepsilon}(\tau) \mathrm{d} \tau=\int_{\varepsilon}^{t_{\varepsilon}} q_{n}^{\prime \prime}(t-\tau) \mathrm{d} \tau+\int_{\varepsilon}^{t_{\varepsilon}} q_{n}^{\prime \prime}(t-\tau)\left(\frac{\rho_{\varepsilon}(\tau)}{\tau}-1\right) \mathrm{d} \tau .
$$

The first integral of the right-hand side is equal to $q_{n}^{\prime}(t-\varepsilon)=q_{n}^{\prime}(t)+O(\varepsilon)$ whereas the second one is $O(\varepsilon)$ since $\left|\rho_{\varepsilon}(\tau) / \tau-1\right| \leq \varepsilon^{2} / \tau^{2}$. This gives the asymptotic behaviour of $\partial u_{n} / \partial t$.

We proceed similarly for $\partial u_{n} / \partial \nu$ starting from an expression of $u_{n}(x, t)$ similar to (33) (with $q_{n}^{\prime}$ and $q_{n}^{\prime \prime}$ replaced by $q_{n}$ and $q_{n}^{\prime}$, respectively). Taking the normal derivative and using again an integration by parts, it is easily seen that

$$
\frac{\partial u_{n}}{\partial \nu}(x, t)=\frac{\varepsilon}{2 \pi} \int_{\varepsilon}^{t_{\varepsilon}}\left(\frac{3 q_{n}(t-\tau)}{\tau^{4}}+\frac{3 q_{n}^{\prime}(t-\tau)}{\tau^{3}}+\frac{q_{n}^{\prime \prime}(t-\tau)}{\tau^{2}}\right) \rho_{\varepsilon}(\tau) \mathrm{d} \tau .
$$

By the same argument as above, the last term in the integral yields a contribution $O(\varepsilon|\log \varepsilon|)$ whereas the second one is $O(1)$. The dominant contribution is given by the first term. Indeed we have

$$
\int_{\varepsilon}^{t_{\varepsilon}} \frac{3 q_{n}(t-\tau)}{\tau^{4}} \rho_{\varepsilon}(\tau) \mathrm{d} \tau=q_{n}(t) \int_{\varepsilon}^{t_{\varepsilon}} \frac{3 \rho_{\varepsilon}(\tau)}{\tau^{4}} \mathrm{~d} \tau+\int_{\varepsilon}^{t_{\varepsilon}} \frac{3\left(q_{n}(t-\tau)-q_{n}(t)\right)}{\tau^{4}} \rho_{\varepsilon}(\tau) \mathrm{d} \tau,
$$


where the last integral is $O\left(\varepsilon^{-1}\right)$ (since $\left|q_{n}(t-\tau)-q_{n}(t)\right| \leq C \tau$ for some $C>0$ ) and

$$
\int_{\varepsilon}^{t_{\varepsilon}} \frac{3 \rho_{\varepsilon}(\tau)}{\tau^{4}} \mathrm{~d} \tau=\int_{\varepsilon}^{t_{\varepsilon}} \frac{\mathrm{d}}{\mathrm{d} t}\left(\frac{\rho_{\varepsilon}(\tau)^{3}}{\varepsilon^{2} \tau^{3}}\right) \mathrm{d} \tau=\frac{1}{\varepsilon^{2}}+O(1)
$$

This completes the proof.

Proof of Proposition 5. From (30) and the definition (29) of the energy flux, the energy conservation law (32) writes as

$$
\begin{aligned}
\mathcal{E}_{\Omega(\varepsilon)}(t) & =\sum_{n, m, p=1}^{N} \mathcal{E}_{n m}^{p}(t, \varepsilon) \quad \text { where } \\
\mathcal{E}_{n m}^{p}(t, \varepsilon) & :=\int_{0}^{t} \int_{\partial B_{p}(\varepsilon)} \frac{\partial u_{n}}{\partial \nu}(x, s) \frac{\partial u_{m}}{\partial t}(x, s) \mathrm{d} \gamma_{x} \mathrm{~d} s .
\end{aligned}
$$

Lemma 7 shows that if $p \neq n$ and $p \neq m$, then $\mathcal{E}_{n m}^{p}(t, \varepsilon)=O\left(\varepsilon^{d-1}\right)$. Moreover if $n \neq m$, then $\mathcal{E}_{n m}^{m}(t, \varepsilon)=$ $O(\varepsilon)$ if $d=3$ (respectively, $\mathcal{E}_{n m}^{m}(t, \varepsilon)=O(\varepsilon|\log \varepsilon|)$ if $d=2$ ) whereas

$$
\mathcal{E}_{n m}^{n}(t, \varepsilon)=\int_{0}^{t} q_{n}(s) \frac{\partial u_{m}}{\partial t}\left(x_{n}, s\right) \mathrm{d} s+O(\varepsilon) .
$$

Finally, if $n=m$, in the three-dimensional case, we have

$$
\begin{aligned}
\mathcal{E}_{n n}^{n}(t, \varepsilon) & =\int_{0}^{t}\left(\frac{q_{n}(s) q_{n}^{\prime}(s)}{4 \pi \varepsilon}-\frac{q_{n}(s) q_{n}^{\prime \prime}(s)}{4 \pi}+o(1)\right) \mathrm{d} s \\
& =\frac{q_{n}(t)^{2}}{8 \pi \varepsilon}+\int_{0}^{t} \frac{q_{n}^{\prime}(s)^{2}}{4 \pi} \mathrm{d} s-\frac{q_{n}(t) q_{n}^{\prime}(t)}{4 \pi}+o(1) .
\end{aligned}
$$

Thus we see that for all time $t$ such that $q_{n}(t) \neq 0$, this quantity diverges as $O\left(\varepsilon^{-1}\right)$ when $\varepsilon$ tends to 0 . On the other hand, when $q_{n}(t)=0$, it has a finite limit given by the second term.

Similarly, in the two-dimensional case, Lemma 7 shows that

$$
\mathcal{E}_{n n}^{n}(t, \varepsilon)=\int_{\varepsilon}^{t} \int_{\varepsilon}^{s} \frac{q_{n}^{\prime}(s-\tau) q_{n}(s)}{2 \pi \tau^{2}} \rho_{\varepsilon}(\tau) \mathrm{d} \tau \mathrm{d} s+\int_{0}^{t} \frac{q_{n}^{\prime}(s) q_{n}(s)}{2 \pi} \mathrm{d} s+O(\varepsilon|\log \varepsilon|) .
$$

The second integral of the right-hand side is equal to $q_{n}(t)^{2} /(4 \pi)$. The first one can be rewritten as

$$
\int_{\varepsilon}^{t}\left(\int_{\tau}^{t}\left(q_{n}^{\prime}(s-\tau)-q_{n}^{\prime}(s)\right) q_{n}(s) \mathrm{d} s+\frac{q_{n}(t)^{2}-q_{n}(\tau)^{2}}{2}\right) \frac{\rho_{\varepsilon}(\tau)}{2 \pi \tau^{2}} \mathrm{~d} \tau
$$

Hence this quantity diverges as $O(|\log \varepsilon|)$ if $q_{n}(t) \neq 0$, since

$$
\int_{\varepsilon}^{t} \frac{\rho_{\varepsilon}(\tau)}{\tau^{2}} \mathrm{~d} \tau=\int_{\varepsilon}^{t} \frac{\mathrm{d}}{\mathrm{d} t}\left(-\frac{\rho_{\varepsilon}(\tau)}{\tau}+\log \left(\rho_{\varepsilon}(\tau)+\tau\right)\right) \mathrm{d} \tau=-\log \varepsilon+O(1)
$$

On the other hand, when $q_{n}(t)=0$, Lebesgue's dominated convergence theorem shows that it tends to

$$
\int_{0}^{t}\left(\int_{\tau}^{t}\left(q_{n}^{\prime}(s-\tau)-q_{n}^{\prime}(s)\right) q_{n}(s) \mathrm{d} s-\frac{q_{n}(\tau)^{2}}{2}\right) \frac{\mathrm{d} \tau}{2 \pi \tau},
$$

which can be rewritten in the simpler form shown in the proposition. 


\section{References}

[1] X. Antoine, B. Pinçon, K. Ramdani, B. Thierry, Far field modeling of electromagnetic time reversal and application to selective focusing on small scatterers, SIAM J. Appl. Math. 69 (2008) 830-844.

[2] C. Ben Amar, N. Gmati, C. Hazard, K. Ramdani, Numerical simulation of time-harmonic reversal mirrors for acoustic waves, SIAM J. Appl. Math. 67 (2007) 777-791.

[3] G. Bal, L. Ryzhik, Time reversal and refocusing in random media, SIAM J. Appl. Math. 63 (2003) 1475-1498.

[4] L. Borcea, G. Papanicolaou, C. Tsogka, J. Berryman, Imaging and time reversal in random media, Inverse Problems 18 (2002) 1247-1279.

[5] L. Borcea, G. Papanicolaou, C. Tsogka, Theory and applications of time reversal and interferometric imaging, Inverse Problems 19 (2003) S134-S164.

[6] M. Born, E. Wolf, Principles of Optics, Cambridge University Press, 1999.

[7] M. Cassier, C. Hazard, Multiple scattering of acoustic waves by small sound-soft obstacles in two dimensions: mathematical justification of the Foldy-Lax model, Wave Motion 50 (2013) 18-28.

[8] R. Elaloufi, R. Carminati, J.-J. Greffet, Diffusive-to-ballistic transition in dynamic light transmission through thin scattering slabs: a radiative transfer approach, J. Opt. Soc. Am. A 21 (2004) 1430-1437.

[9] M. Fink, Acoustic time-reversal mirrors, in: Imaging of Complex Media with Acoustic and Seismic Waves (eds. M. Fink et al), Topic in Applied Physics 84, Springer-Verlag, Berlin (2002) 17-42.

[10] L. L. Foldy, The multiple scattering of waves. I. General theory of isotropic scattering by randomly distributed scatterers, Phys. Rev. 67 (1945) 107-119.

[11] J. Garnier, G. Papanicolaou, Travel time estimation by cross correlation of noisy signals, ESAIM Proc. 27 (2009) 138-135.

[12] C. Hazard, K. Ramdani, Selective acoustic focusing using time-harmonic reversal mirrors, SIAM J. Appl. Math. 64 (2004) 1057-1076.

[13] R. A. Horn, C. A. Johnson, Matrix Analysis, Cambridge University Press, 1985.

[14] T. Kato, Perturbation Theory for Linear Operators, Springer-Verlag, Berlin, 1976.

[15] P. A. Martin, Multiple Scattering. Interaction of Time-Harmonic Waves with N Obstacles, Encyclopedia of Mathematics and its Applications 107, Cambridge University Press, 2006.

[16] E. A. Marengo, F. K. Gruber, Subspace-based localization and inverse scattering of multiply scattering point targets, EURASIP Journal on Advances in Signal Processing (2007) 017342.

[17] N. Mordant, C. Prada, M. Fink, Highly resolved detection and selective focusing in a waveguide using the D.O.R.T. method, J. Acoust. Soc. Am. 105 (1999) 2634-2642.

[18] F. D. Philippe, C. Prada, D. Clorennec, M. Fink, T. Folégot, Construction of the temporal invariants of the time-reversal operator, J. Acoust. Soc. Am. 126 (2009) 8-13.

[19] C. Prada, Detection and imaging in complex media with the D.O.R.T. method, in: Imaging of Complex Media with Acoustic and Seismic Waves (eds. M. Fink et al), Topic in Applied Physics 84, Springer-Verlag (2002) 107-134.

[20] C. Prada, Mémoire d'Habilitation à Diriger les Recherches (French), Université Paris 7, Unpublished, 2006.

[21] C. Prada, M. Fink, Eigenmodes of the time reversal operator: a solution to selective focusing in multiple-target media, Wave Motion 20 (1994) 151-163. 
[22] C. Prada, S. Manneville, D. Spoliansky, M. Fink, Decomposition of the time reversal operator: detection and selective focusing on two scatterers, J. Acoust. Soc. Am. 99 (1996) 2067-2076.

[23] L. N. Trefethen, M. Embree, Spectra and Pseudospectra, Princeton University Press, 2005. 